\title{
STUDY OF AN ASYMPTOTIC PRESERVING SCHEME FOR THE QUASI NEUTRAL EULER-BOLTZMANN MODEL IN THE DRIFT REGIME
}

\author{
MEHDI BADSI ${ }^{1, *}$
}

\begin{abstract}
We deal with the numerical approximation of a simplified quasi neutral plasma model in the drift regime. Specifically, we analyze a finite volume scheme for the quasi neutral Euler-Boltzmann equations. We prove the unconditional stability of the scheme and give some bounds on the numerical approximation that are uniform in the asymptotic parameter. The proof relies on the control of the positivity and the decay of a discrete energy. The severe non linearity of the scheme being the price to pay to get the unconditional stability, to solve it, we propose an iterative linear implicit scheme that reduces to an elliptic system. The elliptic system enjoys a maximum principle that enables to prove the conservation of the positivity under a CFL condition that does not involve the asymptotic parameter. The linear $L^{2}$ stability analysis of the iterative scheme shows that it does not request the mesh size and time step to be smaller than the asymptotic parameter. Numerical illustrations are given to illustrate the stability and consistency of the scheme in the drift regime as well as its ability to compute correct shock speeds.
\end{abstract}

Mathematics Subject Classification. 65M08, 65M06, 65M12, 65Z05.

Received December 4, 2017. Accepted November 8, 2018.

\section{INTRODUCTION}

This work is devoted to the construction of a numerical scheme for the simulation of the quasi-neutral EulerBoltzmann system: such a model represents the evolution of an unmagnetized quasi-neutral plasma where electrons are adiabatic and their density obeys the Boltzmann relation [14,24] and ions are submitted to the self-generated electrostatic field. In this model, we assume that the ions internal energy and the electric energy are of the same order as $\varepsilon^{-1}$ where $\varepsilon>0$ is a possibly small parameter. When $\varepsilon \rightarrow 0$, we reach an asymptotic regime which is called the drift regime: in this regime the pressure force is balanced by the electrostatic-field. In return, the momentum equation for the ions degenerates into an equation where the velocity can be seen as the Lagrange multiplier of the zero total force equation $[6,7,25,28]$. In many practical applications however, the value of $\varepsilon$ is not uniform and can vary with respect to the spatial localization of the plasma. Typically, bounded plasmas are usually such that in the core plasma the regime is $\varepsilon \ll 1$ while near the boundaries, boundary effects such as the Debye sheath may yield a regime where $\varepsilon=\mathcal{O}(1)[2,4,30]$. It is therefore of interest to develop numerical scheme that are able to simulate both regimes. A standard approach to adress this diffuculty consists in a domain decomposition with respect to the local value of $\varepsilon$ : with the use of either the $\varepsilon$-dependent model

Keywords and phrases. Euler-Boltzmann, drift regime, quasi-neutral plasma, asymptotic preserving scheme, stability.

1 Université Paul Sabatier, Institut de Mathématiques de Toulouse (CNRS UMR 5219), France.

*Corresponding author: mehdi.badsi@gmail.com 
in region where $\varepsilon=\mathcal{O}(1)$ or a limit model in regions where $\varepsilon \rightarrow 0$. The coupling of these models is however not straightforward and brings many other technical difficulties related to the interfaces reconnection. To avoid such difficulties, we employ the so called asymptotic preserving approach introduced by Jin $[16,17]$ so as to be able to simulate both regimes $\varepsilon=\mathcal{O}(1)$ and $\varepsilon \rightarrow 0$ with a single model. The efficiency of this approach has led to significant development both in plasma physics and fluid mechanics $[7,8,10,26,28,31]$. However efficient in practice, rigorous stability analysis and proof of convergence in the limit $\varepsilon \rightarrow 0$ for complete physical models is often a difficult task. In this respect, the present work is devoted to analyse some properties of a scheme for the quasi-neutral Euler-Boltzmann system. The motivation of this work comes from some of the questions raised in a previous work of Deluzet et al. [7] on the Euler-Lorentz model. Notably, besides the ability of the scheme to be numerically asymptotic preserving, some questions around the ability of the scheme to preserve the invariants (physical energy, positivity) and computing the correct shock speed in a non conservative form of the Euler-Lorentz equations was formulated.

This work is organized as follows. In Section 2, we introduce the quasi-neutral Euler-Boltzmann system and describe its mathematical properties. In Section 3, we discuss numerical issues related to the Euler-Boltzmann system. In Section 4, we introduce the discretization and define the non linear implicit scheme. Section 4.1 is devoted to prove the existence of a solution to the scheme, its unconditional stability and give some uniform (with respect to the small parameter) bounds. In Section 5, we introduce an iterative linear scheme to solve the non linear scheme. It is proven to be positivity preserving and linear $L^{2}$ stable under a CFL condition that does not involve the small parameter $\varepsilon$. In Section 6 , we illustrate the ability of the scheme to capture the correct shock speeds and test its stability properties in the drift regime.

\section{A FLUID MODEL FOR A QUASI-NEUTRAL PLASMA DYNAMIC: THE SCALED EULER-BOLTZMANN SYSTEM}

We consider a confined plasma that is made of one species of ions. To model this plasma, we use a fluid approach where the plasma position is denoted $x$ and belongs to the unit periodic segment $[0,1]_{\text {per }}=\mathbb{R} / \mathbb{Z}$. In our fluid description, the unknowns of the model are the ions density $n:(t, x) \in[0, T) \times[0,1]_{\text {per }} \rightarrow \mathbb{R}^{+}$, the macroscopic ions velocity $u:(t, x) \in[0, T) \times[0,1]_{\text {per }} \rightarrow \mathbb{R}$, the ions pressure $p:(t, x) \in[0, T) \times[0,1]_{\text {per }} \rightarrow$ $\mathbb{R}^{+}$, and the ions fluid energy density $w:(t, x) \in[0, T) \times[0,1]_{\text {per }} \rightarrow \mathbb{R}^{+}$and the electrostatic potential $\phi:[0, T) \times[0,1]_{\text {per }} \rightarrow \mathbb{R}$. They are assumed to be solutions to the following Euler-Lorentz equations posed in $(0, T) \times[0,1]_{\mathrm{per}}:$

$$
\begin{aligned}
\partial_{t} n+\partial_{x}(n u) & =0, \\
\partial_{t}(n u)+\partial_{x}\left(n u^{2}\right)+\frac{1}{\varepsilon} \partial_{x} p & =\frac{1}{\varepsilon} n E, \\
\partial_{t} w+\partial_{x}(u(w+p)) & =n u E, \\
E & =-\partial_{x} \phi, \\
w & =\frac{\varepsilon n u^{2}}{2}+\frac{1}{\gamma-1} p .
\end{aligned}
$$

Here, the constant parameters $\varepsilon>0$ and $\gamma>1$ denote respectively the thermal energy relatively to the ions kinetic energy and the adiabatic constant. As it is, the system (2.1)-(2.5) is not a closed set of equations, we therefore use the physical hypothesis of quasi-neutrality with adiabatic electrons [24]. Namely, it mathematically writes

$$
\forall(t, x) \in[0, T) \times[0,1], \quad n(t, x)=\mathrm{e}^{\frac{\phi(t, x)}{T_{e}}} \text { with } T_{e}>0 \text { a fixed parameter. }
$$

The set of equations (2.1)-(2.6) now provides a closed system for $(n, u, w, p, \phi)$. The hypothesis of quasineutrality with adiabatic electrons enables to reduce the number of unknowns of the system and to re-write it into a set of conservation laws. Indeed, the algebraic relation (2.6) enables to eliminate the electrostatic potential 
thanks to the following formal computations: from (2.6) we get, $\phi(t, x)=T_{e} \ln (n(t, x))$ and from (2.4) we have $E(t, x)=-T_{e} \frac{\partial_{x} n(t, x)}{n(t, x)}$. Therefore the momentum equation writes in the conservative form:

$$
\partial_{t}(n u)+\partial_{x}\left(n u^{2}\right)+\frac{1}{\varepsilon} \partial_{x}\left(p+T_{e} n\right)=0 .
$$

Some algebra also enables to write the energy equation (2.3) into the conservative form:

$$
\partial_{t}\left(w+T_{e} n(\ln (n)-1)\right)+\partial_{x}\left(u\left(w+p+T_{e} n \ln (n)\right)\right)=0 .
$$

It stems from writing the right hand side of (2.3) into the sum of a temporal and spatial derivate:

$$
n u E=-T_{e} n u \frac{\partial_{x} n}{n}=-T_{e} n u \partial_{x} \ln (n)=-T_{e} \partial_{x}(n u \ln (n))+T_{e} \partial_{x}(n u) \ln (n),
$$

then using the equation (2.1) we obtain:

$$
n u E=-T_{e} \partial_{x}(n u \ln (n))-T_{e} \partial_{t}(n) \ln (n)=-\partial_{t}\left(T_{e} n(\ln (n)-1)\right)-T_{e} \partial_{x}(n u \ln (n))
$$

which is enough to get the conservative energy equation (2.8). Finally, (2.1)-(2.6) is formally equivalent to the following system of conservation laws of unknown $(n, u, w, p, \phi)$ :

$$
\left(P_{\varepsilon}\right):\left\{\begin{array}{l}
\partial_{t} n+\partial_{x}(n u)=0 \\
\partial_{t}(n u)+\partial_{x}\left(n u^{2}\right)+\frac{1}{\varepsilon} \partial_{x}\left(p+T_{e} n\right)=0 \\
\partial_{t}\left(w+T_{e} n(\ln (n)-1)\right)+\partial_{x}\left(u\left(w+p+T_{e} n \ln (n)\right)\right)=0, \\
w=\frac{\varepsilon n u^{2}}{2}+\frac{1}{\gamma-1} p \\
\phi=T_{e} \ln (n) .
\end{array}\right.
$$

The three first equations of $\left(P_{\varepsilon}\right)$ stands for the local conservation of mass, momentum and energy while the two last algebraic equations are constitutive relations relating pressure and electrostatic potential to energy and density. The system $\left(P_{\varepsilon}\right)$ is supplemented with a periodic initial data $\left(n^{0}, u^{0}, w^{0}, p^{0}, \phi^{0}\right)$ where $p^{0}$ and $\phi^{0}$ obey the constitutive relations.

\subsection{Conservation properties and admissible set}

Smooth periodic solutions to $\left(P_{\varepsilon}\right)$ system conserve mass, momentum and energy as stated precisely in the following proposition.

Proposition 2.1. Let $\varepsilon>0$ and $\left(n_{\varepsilon}, u_{\varepsilon}, w_{\varepsilon}, p_{\varepsilon}, \phi_{\varepsilon}\right)$ a smooth periodic solution to $\left(P_{\varepsilon}\right)$ on $[0, T]$. Then one has for all $t \in[0, T)$ :

$$
\begin{array}{r}
\frac{\mathrm{d}}{\mathrm{d} t} \int_{0}^{1} n_{\varepsilon}(t, x) \mathrm{d} x=0, \\
\frac{\mathrm{d}}{\mathrm{d} t} \int_{0}^{1}\left(n_{\varepsilon} u_{\varepsilon}\right)(t, x) \mathrm{d} x=0, \\
\frac{\mathrm{d}}{\mathrm{d} t} \int_{0}^{1}\left(w_{\varepsilon}+T_{e} n_{\varepsilon}\left(\ln \left(n_{\varepsilon}\right)-1\right)\right)(t, x) \mathrm{d} x=0 .
\end{array}
$$

It is also mandatory that solutions to $\left(P_{\varepsilon}\right)$ belong to the admissible set $\Omega=\left\{(n, u, w, p, \phi) \in \mathbb{R}^{5}: n>\right.$ $\left.0, w-\frac{\varepsilon n u^{2}}{2} \geq 0\right\}$. When designing a numerical scheme, it is important to check that the numerical approximation shares the same conservation properties as solutions to the continuous problem. It is a necessary step to assess the robustness of the numerical scheme and often a necessary step towards theoretical convergence result. Significant efforts have been made in this direction in the context of fluid dynamics, for example with the work of Herbin $[12,27]$. 


\subsection{Existence theory for $\left(\boldsymbol{P}_{\varepsilon}\right)$}

The system $\left(P_{\varepsilon}\right)$ can be re-cast into a system of conservation laws for the variables $\left(n, n u, w+T_{e} n(\ln (n)-1)\right)$. It has the form:

$$
\left\{\begin{array}{l}
\partial_{t} W+\partial_{x} f_{\varepsilon}(W)=0, \quad(t, x) \in(0, T) \times[0,1]_{\mathrm{per}} \\
W=(n, q, e)^{t} \text { where } q:=n u, e:=w+T_{e} n(\ln (n)-1) \\
p=(\gamma-1)\left(e-\frac{\varepsilon q^{2}}{2 n}-T_{e} n(\ln (n)-1)\right), \phi=T_{e} \ln (n)
\end{array}\right.
$$

where the flux is defined by

$$
f_{\varepsilon}(W):=\left(\begin{array}{c}
q \\
\frac{q^{2}}{n}+\frac{(\gamma-1)}{\varepsilon}\left(e-\frac{\varepsilon q^{2}}{2 n}-T_{e} n \ln (n)\right)+\frac{\gamma T_{e} n}{\varepsilon} \\
\frac{q}{n}\left(e+(\gamma-1)\left(e-\frac{\varepsilon q^{2}}{2 n}-T_{e} n \ln (n)\right)+\gamma T_{e} n\right)
\end{array}\right) .
$$

Therefore $\left(P_{\varepsilon}\right)$ is strictly hyperbolic since the Jacobian of the flux $f_{\varepsilon}$ has three real distincts eigen values: $\lambda_{-}=u-c_{\varepsilon}<\lambda_{0}=u<\lambda_{+}=u+c_{\varepsilon}$ where

$$
c_{\varepsilon}=\sqrt{\frac{\gamma p / n+\gamma T_{e}}{\varepsilon}}
$$

is the acoustic speed. In the case $T_{e}=0$, the system is nothing but the compressible Euler equations for which existence theory is available $[9,22,29]$. The case $T_{e}>0$ should not rise specific difficulties since considering the specific entropy $S(n, q, e)=\ln \left(\frac{p}{n^{\gamma}}\right)$ the system $\left(P_{\varepsilon}\right)$ admits the additional conservation laws for any $h \in C^{1}(\mathbb{R})$ and smooth solutions:

$$
\partial_{t}(n h(S))+\partial_{x}(n u h(S))=0 .
$$

Particularly, if $h \in C^{2}(\mathbb{R})$ satisfies the inequality $\frac{h^{\prime \prime}(S)}{h^{\prime}(S)}<\frac{1}{\gamma}$ the couple $(-n h(S(n, q, e)),-n u h(S(n, q, e)))$ is an entropy-flux pair and therefore the system $\left(P_{\varepsilon}\right)$ is symmetrizable which enables to apply the Kato-Friedrichs theory [18].

\subsection{The drift limit $\varepsilon \rightarrow 0^{+}$}

The asymptotic $\varepsilon \rightarrow 0^{+}$is called the drift limit. In this limit, the pressure force balances the electric-field. To be more precise, let us assume that smooth solutions to $\left(P_{\varepsilon}\right)$ admits the formal asymptotic Hilbert expansion:

$$
\begin{aligned}
& n_{\varepsilon}=n_{0}+\varepsilon n_{1}+\mathcal{O}\left(\varepsilon^{2}\right), \\
& u_{\varepsilon}=u_{0}+\varepsilon u_{1}+\mathcal{O}\left(\varepsilon^{2}\right), \\
& w_{\varepsilon}=w_{0}+\varepsilon w_{1}+\mathcal{O}\left(\varepsilon^{2}\right) .
\end{aligned}
$$

with $w_{\varepsilon}=\frac{\varepsilon n_{\varepsilon} u_{\varepsilon}^{2}}{2}+\frac{1}{\gamma-1} p_{\varepsilon}$. Plugging this expansion into (2.1)-(2.7) and balancing the equal order terms in $\varepsilon$, we get the following system:

$$
\left\{\begin{array}{l}
\partial_{t} n_{0}+\partial_{x}\left(n_{0} u_{0}\right)=0, \\
\partial_{t}\left(n_{0} u_{0}\right)+\partial_{x}\left(n_{0} u_{0}^{2}\right)+\partial_{x}\left(p_{1}+T_{e} n_{1}\right)=0, \\
\partial_{x}\left(p_{0}+T_{e} n_{0}\right)=0, \\
\partial_{t}\left(w_{0}+T_{e} n_{0}\left(\ln \left(n_{0}\right)-1\right)\right)+\partial_{x}\left(u_{0}\left(w_{0}+p_{0}+T_{e} n_{0} \ln \left(n_{0}\right)\right)\right)=0, \\
w_{0}=\frac{1}{\gamma-1} p_{0}, \\
\phi_{0}=T_{e} \ln \left(n_{0}\right),
\end{array}\right.
$$


Substituting $w_{0}$ in the in the fourth equation and expanding the computation leads to an equation for the pressure that writes:

$$
\partial_{t} p_{0}+\partial_{x}\left(u_{0} p_{0}\right)+(\gamma-1) p_{0} \partial_{x} u_{0}=0
$$

Therefore the formal limit system reads:

$$
\left(P_{0}\right):\left\{\begin{array}{l}
\partial_{t} n_{0}+\partial_{x}\left(n_{0} u_{0}\right)=0 \\
\partial_{t}\left(n_{0} u_{0}\right)+\partial_{x}\left(n_{0} u_{0}^{2}\right)+\partial_{x} \pi_{1}=0 \\
\partial_{x}\left(p_{0}+T_{e} n_{0}\right)=0 \\
\partial_{t} p_{0}+\partial_{x}\left(u_{0} p_{0}\right)+(\gamma-1) p_{0} \partial_{x} u_{0}=0
\end{array}\right.
$$

where $\pi_{1}=p_{1}+T_{e} n_{1}$ is the first order correction of the total pressure. The system $\left(P_{0}\right)$ of unknown $\left(n_{0}, u_{0}, p_{0}, \pi\right)$ is closed. The study of the convergence of the solutions to $\left(P_{\varepsilon}\right)$ toward the solutions of $\left(P_{0}\right)$ when $\varepsilon \rightarrow 0^{+}$is a classical problem arising in fluid mechanics and more generally in the framework of the so called singular limits of hyperbolic systems $[1,19,23]$.

\subsection{Non conservative formulation $\left(P_{\varepsilon}^{\prime}\right)$}

It is sometimes easier to work with a non conservative formulation, that is with a set of variables that is not conserved. As far as the system $\left(P_{\varepsilon}\right)$ is concerned, the energy equation (2.3) can be replaced by an equation on the pressure. Indeed, if we assume a smooth solution $(n, u, w, p, \phi)$ and then multiply the momentum equation (2.2) by $u$, we get:

$$
\left(\partial_{t}(n u)+\partial_{x}\left(n u^{2}\right)+\frac{1}{\varepsilon} \partial_{x}\left(p+T_{e} n\right)\right) u=0 \Rightarrow \partial_{t}\left(n u^{2}\right)+\partial_{x}\left(n u^{3}\right)+\frac{1}{\varepsilon} \partial_{x}\left(p+T_{e} n\right) u=n u \partial_{t} u+n u^{2} \partial_{x} u .
$$

Then thanks to the momentum equation (2.7) and the continuity equation (2.1) one has for the right hand side of the second equation:

$$
n u \partial_{t} u+n u^{2} \partial_{x} u=u\left(n \partial_{t} u+n u \partial_{x} u\right)=u\left(\partial_{t}(n u)+\partial_{x}\left(n u^{2}\right)-u\left(\partial_{t} n+\partial_{x}(n u)\right)\right)=-\frac{u}{\varepsilon} \partial_{x}\left(p+T_{e} n\right) .
$$

We therefore deduce:

$$
\partial_{t}\left(\frac{\varepsilon}{2} n u^{2}\right)+\partial_{x}\left(\frac{\varepsilon}{2} n u^{3}\right)+\partial_{x}\left(p+T_{e} n\right) u=0 .
$$

Substracting the last equation to the conservative form of the energy equation (2.8) gives the following equation for the pressure:

$$
\partial_{t} p+\partial_{x}(u p)+(\gamma-1) p \partial_{x} u=-(\gamma-1) T_{e}\left(\partial_{t}(n \ln (n)-n)+\partial_{x}(u n(\ln (n)-1))+n \partial_{x} u\right)=0
$$

where the right hand side of the last equality vanishes thanks to the continuity equation (2.1). Under the smoothness hypothesis, the system $\left(P_{\varepsilon}\right)$ is equivalent to the following non conservative system:

$$
\left(P_{\varepsilon}^{\prime}\right):\left\{\begin{array}{l}
\partial_{t} n+\partial_{x}(n u)=0 \\
\partial_{t}(n u)+\partial_{x}\left(n u^{2}\right)+\frac{1}{\varepsilon} \partial_{x}\left(p+T_{e} n\right)=0, \\
\partial_{t} p+\partial_{x}(u p)+(\gamma-1) p \partial_{x} u=0, \\
w=\frac{\varepsilon n u^{2}}{2}+\frac{1}{\gamma-1} p \\
\phi=T_{e} \ln (n) .
\end{array}\right.
$$

with a periodic initial data $\left(n^{0}, u^{0}, w^{0}, p^{0}, \phi^{0}\right)$ where $w^{0}$ and $\phi^{0}$ obey the constitutive relations.

\section{Numerical issues Related to the USE OF EITHer $\left(P_{\varepsilon}\right)$ OR $\left(P_{\varepsilon}^{\prime}\right)$}

In this section, we briefly discuss the motivation and the numerical issues we care about in this work concerning the numerical simulation of $\left(P_{\varepsilon}\right)$. 


\subsection{The three dimension Euler-Lorentz model as a motivation}

First and foremost, we mention though it is one dimensional in space, our model contains the essential of the numerical difficulties encountered in the numerical simulation of the three dimensional Euler-Lorentz model for strongly magnetized plasmas. Specifically, in the strong magnetic field regime, the parallel (to the magneticfield) momentum equation degenerates into a balance of force between the parallel gradient of pressure and the parallel electric field. In $[7,28]$ asymptotic preserving schemes are proposed for the three dimensional EulerLorentz model. A special care is dedicated to the reformulation of the model so as to ensure that the spatial discretization would yield formally an estimate of the parallel gradient of total pressure that should scale like the ion Larmor radius. This estimate would ensure the asymptotic consistency of the scheme. One can make an analogy with our one dimensional model, to this effect if one assumes the spatial variable $x$ as being the coordinate aligned to the magnetic field. The essential of the discussion in $[7,28]$ is to ensure an estimate of the form $\partial_{x}\left(p+T_{e} n\right)=\mathcal{O}(\varepsilon)$ in some norm. Linear $L^{2}$ stability issues in the asymptotic $\varepsilon \rightarrow 0$ are also discussed.

\subsection{Positivity and asymptotic consistency: use of $\left(P_{\varepsilon}^{\prime}\right)$}

To derive a numerical scheme that is asymptotically consistent, one has to ensure two things at the discrete level:

(a) The positivity of the total pressure $p+T_{e} n$ so as to avoid unphysical gradient.

(b) The estimate $\partial_{x}\left(p+T_{e} n\right)=\mathcal{O}(\varepsilon)$ for some norm.

The use of an equation on the pressure at the continuous level has the advantage to verify a maximum principle, it then gives a hope to ensure the positivity of at the discrete level. As far as the second point is concerned, we mention that it is not mandatory to employ a non conservative form to ensure the formal asymptotic estimate $\partial_{x}\left(p+T_{e} n\right)=\mathcal{O}(\varepsilon)$, it is also possible to ensure it with the conservative system $\left(P_{\varepsilon}\right)$ (see [15] for a proof). Here the non conservative formulation enables to derive an equation on the total pressure $p+T_{e} n$ that is uniformly elliptic in $\varepsilon[5]$.

\subsection{Asymptotic stability: on the necessity to implicit the gradient of total pressure and the divergence of velocity}

It is known that the numerical discretization of hyperbolic systems involving a small parameter may suffer from severe stiffness on the numerical discretization parameter $[13,26]$. A classical approach to overcome this difficulty is to use the so called IMEX splitting schemes $[3,31]$ that is the discrete counterpart of splitting operator techniques: stiff operators are implicit while the a priori non stiff operators are explicit. Here we propose to explain what is the minimal level of implicitness our numerical scheme shall implement to avoid stability problem in the drift regime $\varepsilon \ll 1$. Let us consider a constant solution $\left(n^{0}, u^{0}, w^{0}, p^{0}, \phi^{0}\right)$ to $\left(P_{\varepsilon}^{\prime}\right)$. Let us consider a solution $(n, u, w, p, \phi)$ to $\left(P_{\varepsilon}^{\prime}\right)$ associated with an initial data that is a small perturbation of the constant state. Let then write the solution as:

$$
n=n^{0}+\tilde{n}, u=u^{0}+\tilde{u}, p=p^{0}+\tilde{p}, w=w^{0}+\tilde{w}, \phi=\phi^{0}+\tilde{\phi} .
$$

Neglecting the second order terms in $\left(P_{\varepsilon}^{\prime}\right)$, it yields the following linearized equations:

$$
\begin{aligned}
n^{0} \partial_{t} \tilde{u}+u^{0}\left(\partial_{t} \tilde{n}+u^{0} \partial_{x} \tilde{n} \partial_{x} \tilde{n}+n^{0} \partial_{x} \partial_{x} \tilde{u}\right)+n^{0} u^{0} \partial_{x} \tilde{u}+\frac{1}{\varepsilon} \partial_{x}\left(\tilde{p}+T_{e} \tilde{n}\right) & =0, \\
\partial_{t} \tilde{p}+u^{0} \partial_{x} \tilde{p}+\gamma p^{0} \partial_{x} \tilde{u} & =0 .
\end{aligned}
$$


where the second term of the momentum equation vanishes thanks to the continuity equation, we therefore obtain the linearized equations for $(\tilde{n}, \tilde{u}, \tilde{p}, \tilde{w}, \tilde{\phi})$ :

$$
\left\{\begin{array}{l}
\partial_{t} \tilde{n}+u^{0} \partial_{x} \tilde{n}+n^{0} \partial_{x} \tilde{u}=0, \\
\partial_{t} \tilde{u}+u^{0} \partial_{x} \tilde{u}+\frac{1}{\varepsilon n^{0}} \partial_{x}\left(\tilde{p}+T_{e} \tilde{n}\right)=0, \\
\partial_{t} \tilde{p}+u^{0} \partial_{x} \tilde{p}+\gamma p^{0} \partial_{x} \tilde{u}=0 \\
\tilde{w}=\varepsilon n^{0} \tilde{u}+\frac{\varepsilon \tilde{n}\left(u^{0}\right)^{2}}{2}+\frac{1}{\gamma-1} \tilde{p} \\
\tilde{\phi}=T_{e} \frac{\tilde{n}}{n^{0}}
\end{array}\right.
$$

To now identify which terms are needed to be implicit, we consider a semi-discretization in time of the form:

$$
\left\{\begin{array}{l}
\forall k \in\left\{0, \ldots,\left\lfloor\frac{T}{\Delta t}\right\rfloor\right\}, \\
\frac{\tilde{n}^{k+1}-\tilde{n}^{k}}{\Delta^{t} t}+u^{0} \partial_{x} \tilde{n}^{k}+n^{0} \partial_{x} \tilde{u}^{k *}=0, \\
\frac{\tilde{u}^{k+1}-\tilde{u}^{k}}{\varepsilon u^{0}}+\partial_{x} \tilde{u}^{k}+\frac{1}{\varepsilon n^{0}} \partial_{x}\left(\tilde{p}^{k+1}+T_{e} \tilde{n}^{k+1}\right)=0, \\
\frac{\tilde{p}^{k+1}-\tilde{p}^{k}}{\Delta t}+u^{0} \partial_{x} \tilde{p}^{k}+\gamma p^{0} \partial_{x} \tilde{u}^{k *}=0,
\end{array}\right.
$$

where $\Delta t>0$ and $\tilde{n}^{k}, \tilde{u}^{k}, \tilde{p}^{k}$ stand for an approximation at time $t_{k}=k \Delta t>0$ of $\tilde{n}\left(t^{k},.\right), \tilde{u}\left(t^{k},.\right), \tilde{p}\left(t^{k},.\right)$. If we take $k *=k$, and use the linearized momentum equation to express $\tilde{u}^{k *}$ as a function of $\partial_{x}\left(\tilde{p}^{k}+T_{e} \tilde{n}^{k}\right)$, therefore we obtain by inserting $u^{k *}$ in both the linearized density and pressure equations, an elliptic equation for the total pressure that takes the form:

$$
\tilde{p}^{k+1}+T_{e} \tilde{n}^{k+1}-\frac{\Delta t^{2}}{\varepsilon} \partial_{x x}\left(\tilde{p}^{k}+T_{e} \tilde{n}^{k}\right)=\tilde{r}^{k}
$$

where $\tilde{r}^{k}$ is a residual term.

Such an explicit discretization would yield a stability constrain that is dependent on $\varepsilon$. From a computational point of view, it is unacceptable. However, if we chose $k *=k+1$ the stiff term becomes implicit and the discretization would yield a stability constrain that is dependent on $u^{0}$ but not on $\varepsilon$. In this scope, the minimal level of implicitness is:

(a) To treat the gradient of total pressure in the momentum equation implicitly.

(b) To treat the divergence of velocity in the mass flux and in the pressure equation implicitly.

Let us mention however that the implicitness of the gradient of total pressure is not the only way to ensure stability independently on $\varepsilon$. Another way to do it $[15,26]$, is to split the gradient of pressure $\frac{1}{\varepsilon} \partial_{x}\left(\tilde{p}^{k+1}+T_{e} \tilde{n}^{k+1}\right)$, and to substitute it by $\alpha \partial_{x}\left(\tilde{p}^{k}+T_{e} \tilde{n}^{k}\right)+\left(\frac{1}{\varepsilon}-\alpha\right) \partial_{x}\left(\tilde{p}^{k+1}+T_{e} \tilde{n}^{k+1}\right)$ where $\alpha>0$ is a numerical parameter that must be correctly chosen so as to ensure the stability of the scheme.

\subsection{Shock speed computation for the non conservative formulation $\left(P_{\varepsilon}^{\prime}\right)$ and energy conservation}

Though it is convenient to work with $\left(P_{\varepsilon}^{\prime}\right)$ to ensure the positivity of the pressure (because it is not in this case the difference of two non negative terms) and the formal AP consistency, it brings another difficulty related to the hyperbolic structure of the Euler-Boltzmann model. It is well-known that for hyperbolic systems [20], solutions are rarely classical even if the initial data and the boundary conditions are smooth. Solutions develop discontinuities owing to the fact that characteristics cross in finite time. In this case the pressure equation and the energy equation are not equivalent. Therefore $\left(P_{\varepsilon}^{\prime}\right)$ and $\left(P_{\varepsilon}\right)$ are no longer equivalent. To palliate this eventuality, we use the strategy developed in [27]. We shall add a corrective source term in the discretization of (2.11) that accounts for the numerical representation of measures appearing when the solution is no longer smooth. It is proven in [27] that under suitable a priori estimates, such an approach allows to recover the 
Rankine-Hugoniot relations when the mesh size and time step tend to zero. To clarify the discussion, let us briefly sketch what information is lost in the computation of Section 2.4 when solutions are no longer smooth. Consider the simple inviscid Burgers equation posed in $(0,+\infty) \times \mathbb{R}$ :

$$
\partial_{t} s+\partial_{x}\left(\frac{s^{2}}{2}\right)=0
$$

In the case $s$ is smooth, we can multiply by $s(3.1)$ and perform differentiation in time and space to obtain:

$$
\partial_{t}\left(\frac{s^{2}}{2}\right)+\partial_{x}\left(\frac{s^{3}}{3}\right)=0
$$

Assuming now a solution of the form $s(t, x)=s_{l} \mathbf{1}_{x \leq \sigma t}(t, x)+s_{r} \mathbf{1}_{x>\sigma t}(t, x)$ yields the Rankine-Hugoniot relation for the first equation $\sigma=\frac{s_{l}+s_{r}}{2}$ while for the second $\sigma=\frac{2}{3}\left(s_{l}+s_{r}\right)$. It therefore gives different shock speeds. One may ask what is missing in (3.2) and why do we obtain different shock speeds. To understand, let us write the weak formulation of the Burgers equation (3.1). We shall say that $s \in L^{\infty}((0,+\infty) \times \mathbb{R})$ satisfies the Burgers equation (3.1) in the weak sense if

$$
\int_{\mathbb{R}} \int_{0}^{+\infty} s \partial_{t} \varphi+f(s) \partial_{x} \varphi \mathrm{d} t \mathrm{~d} x=0 \quad \forall \varphi \in \mathcal{C}_{c}^{1}((0,+\infty) \times \mathbb{R})
$$

where $f(s)=\frac{s^{2}}{2}$. To mimick the formal computation of Section 2.4, or simply to try to obtain (3.2), we are tempted to take $\varphi=s \psi$ for some $\psi \in \mathcal{C}_{c}^{1}((0,+\infty) \times \mathbb{R})$. However it is not possible since a priori $s \notin \mathcal{C}^{1}((0,+\infty) \times \mathbb{R})$. Let us proceed by regularization: there is $s_{n} \in \mathcal{D}((0,+\infty) \times \mathbb{R})$ such that $s_{n} \rightarrow s$ in $L_{\text {loc }}^{p}((0,+\infty) \times \mathbb{R})$ for $1 \leq p<\infty$.

Now take $\varphi=s_{n} \psi$ with $\psi \in \mathcal{C}_{c}^{1}((0,+\infty) \times \mathbb{R})$ we obtain:

$$
\int_{\mathbb{R}} \int_{0}^{+\infty} s s_{n} \partial_{t} \psi+f(s) s_{n} \partial_{x} \psi \mathrm{d} t \mathrm{~d} x=-\int_{\mathbb{R}} \int_{0}^{+\infty}\left(s \partial_{t} s_{n}+f(s) \partial_{x} s_{n}\right) \psi \mathrm{d} t \mathrm{~d} x .
$$

It is easy to see that the left hand side tends to $\int_{\mathbb{R}} \int_{0}^{+\infty} s^{2} \partial_{t} \psi+f(s) s \partial_{x} \psi \mathrm{d} t \mathrm{~d} x$ as $n \rightarrow+\infty$. As for the right hand side, if $s \partial_{t} s_{n}+f(s) \partial_{x} s_{n}$ is only bounded in $L_{\text {loc }}^{1}((0,+\infty) \times \mathbb{R})$ then up to a subsequence, $s \partial_{t} s_{n}+f(s) \partial_{x} s_{n}$ tends to a measure. Thus in the case solutions are discontinuous, weak solutions to (3.1) are different from weak solutions to (3.2), they have both different weak formulations. To ensure the consistency between the two formulations at the discrete level, one has to take into account this measure. Our last numerical concern in this work, is the conservation of a discrete analogue of the energy equation (2.3). To obtain the conservation of the energy, one shall perform the formal computation of Section 2.4 in the opposite sense. To do so one has to ensure the discrete analogue of the following properties:

- The cancellation at the discrete level:

$$
\int_{[0,1]_{\mathrm{per}}} \partial_{x}\left(p+T_{e} n\right) u+\left(p+T_{e} n\right) \partial_{x} u \mathrm{~d} x=0 .
$$

- Make a crucial use of the the continuity equation $(2.1): \partial_{t} n+\partial_{x}(n u)=0$.

A convenient tool to ensure these two properties is to use a staggered discretization where density and pressure are discretized on a primal mesh, while the velocity is discretized on a dual mesh. It enables to build discrete differential operator such that the duality between the "divergence" of the velocity and the gradient of total pressure holds. Another important property is that the continuity equation needs to be valid both on the primal and the dual mesh. 


\section{An unconditionally Stable nOn linear implicit SCheme for $\left(P_{\varepsilon}^{\prime}\right)$}

In this section, we introduce the discretization and propose a non linear implicit scheme to approximate solutions to $\left(P_{\varepsilon}^{\prime}\right)$. Let us begin with the mesh notation. Let a time step $\Delta t>0$ and define for all $k \in\left\{0,1, \ldots,\left\lfloor\frac{T}{\Delta t}\right\rfloor\right\}$ the discrete times $t_{k}:=k \Delta t$. Let $N \in \mathbb{N}^{*}$ and $\Delta x:=\frac{1}{N+1}$ be the mesh size. The the periodic unit segment line $[0,1]_{\text {per }}$ is discretized with the points defined for all $i \in \mathbb{Z}$ by $x_{i}:=i \Delta x$ where for all $(i, j) \in \mathbb{Z}^{2}$, the two points $x_{i}$ and $x_{j}$ denote the same grid point and we note it $x_{i} \equiv x_{j}$ if and only if $x_{i}-x_{j} \in \mathbb{Z}$. In particular, we have for all $i \in \mathbb{Z}, x_{i+(N+1)} \equiv x_{i}$. In the spirit of finite volume framework, we introduce the control cell $C_{i}:=\left[x_{i-\frac{1}{2}}, x_{i+\frac{1}{2}}\right)$ where $x_{i-\frac{1}{2}}:=x_{i}-\frac{\Delta x}{2}, \quad x_{i+\frac{1}{2}}:=x_{i}+\frac{\Delta x}{2}$. We also define the cell centered on $x_{i-\frac{1}{2}}$ by $C_{i-\frac{1}{2}}:=\left[x_{i-1}, x_{i}\right)$. We use a staggered discretization, namely the density and pressure are discretized on the primal mesh $\mathcal{T}_{h}:=\cup_{i \in \mathbb{Z}} C_{i}$ while the velocity is discretized on the dual mesh $\mathcal{T}_{h}^{*}:=\cup_{i \in \mathbb{Z}} C_{i-\frac{1}{2}}$. In all the sequel, we shall omit to precise the dependence on $T_{e}>0$ and $\gamma>1$ of the solutions to $\left(P_{\varepsilon}^{\prime}\right)$ for any $\varepsilon>0$. For any $\varepsilon>0$, the discretization of $\left(P_{\varepsilon}^{\prime}\right)$ consists in approaching for each $k \in\left\{0,1, \ldots,\left\lfloor\frac{T}{\Delta t}\right\rfloor\right\}$, the unknown functions $n_{\varepsilon}\left(t^{k},.\right)$, $p_{\varepsilon}\left(t^{k},.\right), u_{\varepsilon}\left(t^{k},.\right)$ by:

$$
\begin{aligned}
& n_{\varepsilon}\left(t^{k}, x\right) \approx n_{\varepsilon, h}^{k}(x):=\sum_{i \in \mathbb{Z}} n_{\varepsilon, i}^{k} \mathbf{1}_{C_{i}}(x), \\
& p_{\varepsilon}\left(t^{k}, x\right) \approx p_{\varepsilon, h}^{k}(x):=\sum_{i \in \mathbb{Z}} p_{\varepsilon, i}^{k} \mathbf{1}_{C_{i}}(x), \\
& u_{\varepsilon}\left(t^{k}, x\right) \approx u_{\varepsilon, h}^{k}(x):=\sum_{i \in \mathbb{Z}} u_{\varepsilon, i-\frac{1}{2}}^{k} \mathbf{1}_{C_{i-\frac{1}{2}}}(x),
\end{aligned}
$$

where the three sequences $\left(n_{\varepsilon, i}^{k}\right)_{i \in \mathbb{Z}} \subset \mathbb{R},\left(p_{\varepsilon, i}^{k}\right)_{i \in \mathbb{Z}} \subset \mathbb{R},\left(u_{\varepsilon, i-\frac{1}{2}}^{k}\right)_{i \in \mathbb{Z}} \subset \mathbb{R}$ are assumed to be a solution to the following non linear scheme $\left(P_{\varepsilon, \Delta t, \Delta x}^{\prime}\right)$ :

$$
\left\{\begin{array}{l}
\forall i \in\{0, \ldots, N\} \\
\frac{\Delta x}{\Delta t}\left(n_{\varepsilon, i}^{k+1}-n_{\varepsilon, i}^{k}\right)+ \\
\frac{\Delta x}{\Delta t}\left(n_{\varepsilon, i-\frac{1}{2}}^{k+1} u_{\varepsilon, i-\frac{1}{2}}^{k+1}\right. \\
\frac{\Delta x}{\Delta t}\left(p_{\varepsilon, i}^{k+1}-p_{\varepsilon, i}^{k}\right)+ \\
\text { with the periodic bo } \\
\forall i \in \mathbb{Z}, \\
n_{\varepsilon, i}^{k+1}=n_{\varepsilon, i+(N+1)}^{k+1}, \\
u_{\varepsilon, i-\frac{1}{2}}^{k+1}=u_{\varepsilon, i+N+\frac{1}{2}}^{k+1}, \\
p_{\varepsilon, i}^{k+1}=p_{\varepsilon, i+(N+1)}^{k+1}
\end{array}\right.
$$

where all terms will be defined here under. The non linear scheme is supplemented with a periodic initial data $\left(n_{\varepsilon, i}^{0}\right)_{i \in \mathbb{Z}} \subset \mathbb{R}_{*}^{+},\left(u_{\varepsilon, i-\frac{1}{2}}^{0}\right)_{i \in \mathbb{Z}} \subset \mathbb{R},\left(p_{\varepsilon, i}^{0}\right)_{i \in \mathbb{Z}} \subset \mathbb{R}^{+}$where for all $i \in \mathbb{Z}$ :

$$
n_{\varepsilon, i}^{0}=\frac{1}{\Delta x} \int_{C_{i}} n_{\varepsilon}^{0}(x) \mathrm{d} x, \quad u_{\varepsilon, i-\frac{1}{2}}^{0}=\frac{1}{\Delta x} \int_{C_{i-\frac{1}{2}}} u_{\varepsilon}^{0}(x) \mathrm{d} x, \quad p_{\varepsilon, i}^{0}=\frac{1}{\Delta x} \int_{C_{i}} p_{\varepsilon}^{0}(x) \mathrm{d} x .
$$

For consiceness, we shall use the notation $\left(n_{\varepsilon}^{k}, p_{\varepsilon}^{k}, u_{\varepsilon}^{k}\right) \in \mathbb{R}^{\mathbb{Z}} \times \mathbb{R}^{\mathbb{Z}} \times \mathbb{R}^{\mathbb{Z}}$ to denote the sequences $\left(n_{\varepsilon, i}^{k}\right)_{i \in \mathbb{Z}} \subset$ $\mathbb{R},\left(p_{\varepsilon, i}^{k}\right)_{i \in \mathbb{Z}} \subset \mathbb{R},\left(u_{\varepsilon, i-\frac{1}{2}}^{k}\right)_{i \in \mathbb{Z}} \subset \mathbb{R}$ at an iteration $k \in \mathbb{N}$. The staggered discretization (4.1)-(4.11) features two important properties: 
(a) For all $k \in\left\{0, \ldots,\left\lfloor\frac{T}{\Delta t}\right\rfloor\right\}$, the duality formula

$$
\int_{[0,1]_{\mathrm{per}}} u_{\varepsilon, h}^{k}(x) \partial_{x} p_{\varepsilon, h}^{k}(x) d x=-\int_{[0,1]_{\mathrm{per}}} p_{\varepsilon, h}^{k}(x) \partial_{x} u_{\varepsilon, h}^{k}(x) d x
$$

holds, where $\partial_{x} p_{\varepsilon, h}^{k}:=\sum_{i \in \mathbb{Z}} \frac{\left(p_{\varepsilon, i+1}^{k}-p_{\varepsilon, i}^{k}\right)}{\Delta x} \mathbf{1}_{C_{i+\frac{1}{2}}}$ and $\partial_{x} u_{\varepsilon, h}^{k}:=\sum_{i \in \mathbb{Z}} \frac{\left(u_{\varepsilon, i+\frac{1}{2}}^{k}-u_{\varepsilon, i-\frac{1}{2}}^{k}\right)}{\Delta x} \mathbf{1}_{C_{i}}$.

(b) The validity of the discrete continuity equation on the dual mesh $\mathcal{T}_{h}^{*}$ :

$$
\forall i \in\{0, \ldots, N\}, \frac{\Delta x}{\Delta t}\left(n_{\varepsilon, i}^{k+1}-n_{\varepsilon, i}^{k}\right)+F_{\varepsilon, i+\frac{1}{2}}^{k+1}-F_{\varepsilon, i-\frac{1}{2}}^{k+1}=0 \Rightarrow \frac{\Delta x}{\Delta t}\left(n_{\varepsilon, i-\frac{1}{2}}^{k+1}-n_{\varepsilon, i-\frac{1}{2}}^{k}\right)+F_{\varepsilon, i}^{k+1}-F_{\varepsilon, i-1}^{k+1}=0 .
$$

where $n_{\varepsilon, i-\frac{1}{2}}^{k}$ and $F_{\varepsilon, i}^{k}$ are defined here under by (4.15) and (4.16).

Now we shall defined precisely, the flux and the density at the interfaces. Our scheme follows the standard finite volumes approach, the discrete continuity (4.5) equation is obtained by integrating the continuity equation (2.1) on $C_{i}$ and considering the discrete values $n_{\varepsilon}^{k}$ as an approximation of the mean values of the density at times $t_{k}$ in the cell $C_{i}$. The flux of mass at the interface is upwind on the density with respect to the sign of the velocity:

$$
F_{\varepsilon, i+\frac{1}{2}}^{k+1}=\left(u_{\varepsilon, i+\frac{1}{2}}^{k+1}\right)^{+} n_{\varepsilon, i}^{k+1}-\left(u_{\varepsilon, i+\frac{1}{2}}^{k+1}\right)^{-} n_{\varepsilon, i+1}^{k+1} .
$$

where $(.)^{+}:=\max (., 0)$ and $(.)^{-}:=\max (-., 0)$ denote respectively the positive and the negative part functions. The discrete momentum equation (4.6) is obtained by integrating momentum equation (2.7) on $C_{i-\frac{1}{2}}$. The density is here defined in the cell $C_{i-\frac{1}{2}}$ as the mean value over the two neighbouring cells:

$$
n_{\varepsilon, i-\frac{1}{2}}^{k}:=\frac{n_{\varepsilon, i-1}^{k}+n_{\varepsilon, i}^{k}}{2} .
$$

The flux of mass and the velocity at the interfaces of the cell $C_{i-\frac{1}{2}}$ are defined by:

$$
F_{\varepsilon, i}^{k}:=\frac{F_{\varepsilon, i-\frac{1}{2}}^{k}+F_{\varepsilon, i+\frac{1}{2}}^{k}}{2}
$$

The velocity is here upwind with respect to the sign of the flux of mass:

$$
u_{\varepsilon, i}^{k}:= \begin{cases}u_{\varepsilon, i-\frac{1}{2}}^{k} & \text { if } F_{\varepsilon, i}^{k} \geq 0 \\ u_{\varepsilon, i+\frac{1}{2}}^{k} \text { otherwise. }\end{cases}
$$

In (4.6), $\delta_{i-\frac{1}{2}}$ denotes the two points finite difference operator centered at $x_{i-\frac{1}{2}}$, namely,

$$
\delta_{i-\frac{1}{2}}\left(p_{\varepsilon}^{k+1}+T_{e} n_{\varepsilon}^{k+1}\right):=\left(p_{\varepsilon, i}^{k+1}-p_{\varepsilon, i-1}^{k+1}\right)+T_{e}\left(n_{\varepsilon, i}^{k+1}-n_{\varepsilon, i-1}^{k+1}\right) .
$$

Finally, the discrete pressure equation is obtained by integrating the pressure equation $(2.11)$ in $C_{i}$. The flux of pressure at the interface is upwind on the pressure with respect to the sign of the velocity:

$$
(u p)_{\varepsilon, i+\frac{1}{2}}^{k+1}:=\left(u_{\varepsilon, i+\frac{1}{2}}^{k+1}\right)^{+} p_{\varepsilon, i}^{k+1}-\left(u_{\varepsilon, i+\frac{1}{2}}^{k+1}\right)^{-} p_{\varepsilon, i+1}^{k+1} .
$$

In the discrete pressure equation $(4.7), \delta_{i}$ denotes the two point finite difference operator centered at $x_{i}$, namely

$$
\delta_{i}\left(u_{\varepsilon}^{k+1}\right):=u_{\varepsilon, i+\frac{1}{2}}^{k+1}-u_{\varepsilon, i-\frac{1}{2}}^{k+1} .
$$


Lastly, the corrective source term is non negative and here defined for all $i \in\{0, \ldots, N\}$ by:

$$
\begin{aligned}
\frac{2}{\varepsilon(\gamma-1)} S_{\varepsilon, i}^{k+1}:= & \frac{\Delta x}{2 \Delta t} n_{\varepsilon, i}^{k}\left(u_{\varepsilon, i+\frac{1}{2}}^{k+1}-u_{\varepsilon, i+\frac{1}{2}}^{k}\right)^{2}+\frac{\Delta x}{2 \Delta t} n_{\varepsilon, i}^{k}\left(u_{\varepsilon, i-\frac{1}{2}}^{k+1}-u_{\varepsilon, i-\frac{1}{2}}^{k}\right)^{2} \\
& +\left|F_{\varepsilon, i}^{k+1}\right|\left(u_{\varepsilon, i+\frac{1}{2}}^{k+1}-u_{\varepsilon, i-\frac{1}{2}}^{k+1}\right)^{2}
\end{aligned}
$$

The source term $S_{\varepsilon, i}^{k+1}$ is a numerical representation of measures appearing when solutions develop discontinuities. It is designed to compensate in the cell $C_{i}$ the contribution of the residual terms $R_{\varepsilon, i-\frac{1}{2}}^{k+1}$ and $R_{\varepsilon, i+\frac{1}{2}}^{k+1}$ that originate from the kinetic energy balance (4.23).

Let us now make some comments on different aspects of the scheme:

- The two properties (4.12) and (4.13) together with the presence of the source term (4.21) are crucial to solve the problem of having a conservative scheme that computes the correct shock speeds (see Sect. 3.4). Specifically, the purpose of the duality formula (4.12) is to ensure the cancellation of the sum of the integral of non conservative products. The validity of the continuity equation on the dual mesh (4.13) aims at recovering a discrete total energy balance: namely, we want to mimic the formal computation of Section 2.4 so as to derive an equation for the kinetic energy (2.8). Altogether, these properties ensure the consistency of the scheme $\left(P_{\varepsilon, \Delta t, \Delta x}^{\prime}\right)$ with $\left(P_{\varepsilon}\right)$ when $\Delta t$ and $\Delta x$ tend to zero. It is necessary, if one wants to recover the Rankine-Hugoniot relations (for further details we refer to [12]). In this respect, we performed discrete time and space differentiation on the discrete momentum equation and continuity equation (4.6), (4.5). Using (4.12) and (4.13), we obtained in Proposition 4.2 a discrete kinetic energy balance with a residual term and in Proposition 4.3 a discrete potential energy balance with another residual term. On the contrary to our formal computation of Section 3.4 where the solution was assumed to be smooth, in Propositions 4.2 and 4.3 the discrete numerical approximation is a priori discontinuous. It thus yields the presence of Dirac measures. The residual terms are therefore the numerical manifestation of this measures. For instance, one can check that if the velocity $u_{\varepsilon}$ has a discontinuity then the residual term in Proposition 4.2 does not tend to zero when $\Delta x$ and $\Delta t$ do. The role of $S_{\varepsilon, i}^{k+1}$ is therefore to compensate the contribution of this residual term on each cell.

- It is proven in Lemmas 2.1 and 3.1 of [11] that such a discretization of the continuity equation and the pressure equation yields the unconditional positivity of the density (provided it is at initial time) and the unconditional non negativity of the pressure (provided it is at initial time). Essentially, the proof relies on the algebraic structure of the discretization of the continuity equation that plays a central role in the derivation of stability estimate. The discrete continuity equation (4.5) is used to prove the non-negativity of the pressure. Without going too much in the details, with the choice of an upwind flux the discrete continuity equation (4.5) can be re-written into the form:

$$
M\left(u_{\varepsilon}^{k+1}\right) n_{\varepsilon}^{k+1}=n_{\varepsilon}^{k}
$$

where $M\left(u^{k+1}\right) \in \mathcal{M}_{N+1}(\mathbb{R})$ is an $M$-matrix. As far as the nonnegativity of the pressure is concerned, the authors in [11] work with the internal energy. One can re-write the discrete pressure equation (4.7) in terms of the internal energy by decomposing the pressure as $p_{\varepsilon, i}^{k}=n_{\varepsilon, i}^{k} s_{\varepsilon, i}^{k}$ and obtain that the pressure equation re-writes

$$
\frac{\Delta x}{\Delta t}\left(n_{\varepsilon, i}^{k+1} s_{\varepsilon, i}^{k+1}-n_{\varepsilon, i}^{k} s_{\varepsilon, i}^{k}\right)+F_{\varepsilon, i+\frac{1}{2}}^{k+1} s_{\varepsilon, i+\frac{1}{2}}^{k+1}-F_{\varepsilon, i-\frac{1}{2}}^{k+1} s_{\varepsilon, i-\frac{1}{2}}^{k+1}+n_{\varepsilon, i}^{k+1}\left(s_{\varepsilon, i}^{k+1}\right)^{+} \delta_{i}\left(u_{\varepsilon}^{k+1}\right)=S_{\varepsilon, i}^{k+1}
$$

where

$$
s_{\varepsilon, i+\frac{1}{2}}^{k+1}=\left\{\begin{array}{l}
s_{\varepsilon, i}^{k+1} \text { if } F_{\varepsilon, i+\frac{1}{2}}^{k+1} \geq 0 \\
s_{\varepsilon, i+1}^{k+1} \text { otherwise }
\end{array}\right.
$$


Multiplying by $\left(s_{\varepsilon, i}^{k+1}\right)^{-}$, using the discrete continuity equation (4.5) and summing enables to show that $\left(s_{\varepsilon, i}^{k}\right)^{-}=0$ for all $i \in\{0, \ldots, N\}$. The discussion of Section 3.3 on the stability shows that to avoid the standard CFL stability condition of the form $\frac{\Delta t}{\Delta x} \underset{\varepsilon \rightarrow 0}{=} \mathcal{O}\left(\varepsilon^{\frac{1}{2}}\right)$, it is necessary to implicit the gradient of total pressure in the momentum equation (2.2) as well as the flux of mass in the continuity equation (2.1). Keeping this in mind, to recover some energy balance, it also requires to implicit the flux of mass also in the momentum equation (2.2). This is why our scheme is fully implicit. It may possible that another approach, as in the spirit of pressure-correction scheme [12] may avoid the implicitness of the flux to obtain stability under a CFL number that does not depend on $\varepsilon$.

- The flux of mass and pressure (4.14)-(4.19) at the interfaces can be written under the form:

$$
\begin{aligned}
F_{\varepsilon, i+\frac{1}{2}}^{k+1} & =n_{\varepsilon, i+\frac{1}{2}}^{k+1} u_{\varepsilon, i+\frac{1}{2}}^{k+1}-\frac{\left(n_{\varepsilon, i+1}^{k+1}-n_{\varepsilon, i}^{k+1}\right)}{2}\left|u_{\varepsilon, i+\frac{1}{2}}^{k+1}\right|, \\
(u p)_{\varepsilon, i+\frac{1}{2}}^{k+1} & =\frac{\left(p_{\varepsilon, i+1}^{k+1}+p_{\varepsilon, i}^{k+1}\right)}{2} u_{\varepsilon, i+\frac{1}{2}}^{k+1}-\frac{\left(p_{\varepsilon, i+1}^{k+1}-p_{\varepsilon, i}^{k+1}\right)}{2}\left|u_{\varepsilon, i+\frac{1}{2}}^{k+1}\right| .
\end{aligned}
$$

which can be seen as a Rusanov flux where the viscosity only depends on the velocity. In the standard Rusanov flux [21], the viscosity depends on the maximal characteristics speeds of the system which in our case would include the acoustic speed $c_{\varepsilon}(2.9)$.

We have the following.

Theorem 4.1 (Positivity). Let $\varepsilon>0$, and let $n_{\varepsilon}^{0} \in\left(\mathbb{R}_{*}^{+}\right)^{\mathbb{Z}}, p_{\varepsilon}^{0} \in\left(\mathbb{R}^{+}\right)^{\mathbb{Z}}, u_{\varepsilon}^{0} \in \mathbb{R}^{\mathbb{Z}}$ periodic. Let $k \in\left\{0, \ldots,\left\lfloor\frac{T}{\Delta T}\right\rfloor\right\}$ and $n_{\varepsilon}^{k} \in \mathbb{R}^{\mathbb{Z}}, u_{\varepsilon}^{k} \in \mathbb{R}^{\mathbb{Z}}, p_{\varepsilon}^{k} \in \mathbb{R}^{\mathbb{Z}}$ a solution to $\left(P_{\varepsilon, \Delta t, \Delta x}^{\prime}\right)$. Therefore for all $i \in \mathbb{Z} n_{\varepsilon, i}^{k}>0$ and $p_{\varepsilon, i}^{k} \geq 0$.

\subsection{Unconditional stability and uniform in $\varepsilon$ bounds}

In this section, we are concerned with the well posedness of the scheme $\left(P_{\varepsilon, \Delta t, \Delta x}^{\prime}\right)$. More precisely, we are going to prove that:

- The scheme is well-defined. Namely it has a solution for any positive numbers $\varepsilon, \Delta t, \Delta x$.

- Solutions to the scheme are unconditionnaly stable. Namely, for any positive numbers $\varepsilon, \Delta t, \Delta x$, the scheme preserves the positivity of the density, the non negativity of the pressure, the total mass, the total momentum. It dissipates some discrete energy functional and provides $L^{\infty}$ bounds on the pressure and density that are uniform in $\varepsilon$ as soon as the initial data are $\mathcal{O}(1)$ as $\varepsilon \rightarrow 0^{+}$.

By periodicity, summing the discrete continuity equation (4.5) and the discrete momentum equation (4.6) yields the conservation of the total mass and momentum, namely for all $k \in\left\{0, \ldots,\left\lfloor\frac{T}{\Delta t}\right\rfloor\right\}$ :

$$
\sum_{i=0}^{N} n_{\varepsilon, i}^{k}=\sum_{i=0}^{N} n_{\varepsilon, i}^{0}, \quad \sum_{i=0}^{N} n_{\varepsilon, i-\frac{1}{2}}^{k} u_{\varepsilon, i-\frac{1}{2}}^{k}=\sum_{i=0}^{N} n_{\varepsilon, i-\frac{1}{2}}^{0} u_{\varepsilon, i-\frac{1}{2}}^{0} .
$$

Moreover, because the scheme also provides the density to be positive, we control the $L^{1}$-norm of the discrete density $n_{\varepsilon, h}^{k}$ and thus any norm since we are working in finite dimensional functional spaces. This therefore gives the $L^{\infty}$ boundedness of the density. The cornerstone to get the existence of a solution to $\left(P_{\varepsilon, \Delta t, \Delta x}\right)^{\prime}$ and an $L^{\infty}$ bound on the pressure, is to obtain an estimate for the discrete energy. More precisely, let us define for a solution $\left(n_{\varepsilon}^{k}, u_{\varepsilon}^{k}, p_{\varepsilon}^{k}\right)$ to the scheme $\left(P_{\varepsilon, \Delta t, \Delta x}^{\prime}\right)$ the discrete energy functional:

$$
\mathcal{E}\left(n_{\varepsilon}^{k}, u_{\varepsilon}^{k}, p_{\varepsilon}^{k}\right)=\sum_{i=0}^{N}\left(\frac{\varepsilon n_{\varepsilon, i-\frac{1}{2}}^{k}\left(u_{\varepsilon, i-\frac{1}{2}}^{k}\right)^{2}}{2}+\frac{p_{\varepsilon, i}^{k}}{\gamma-1}+T_{e} n_{\varepsilon, i}^{k}\left(\ln \left(n_{\varepsilon, i}^{k}\right)-1\right)\right) \Delta x .
$$


To prove the decay of this functional, we need a discrete analogue of the conservative energy equation (2.8). To do so, we crucially uses the duality property (4.12) and the validity of the discrete continuity equation (4.13) on the dual mesh. One has the following.

Proposition 4.2 (Kinetic energy balance). Let $\varepsilon>0$, let $n_{\varepsilon}^{0} \in\left(\mathbb{R}_{*}^{+}\right)^{\mathbb{Z}}, p_{\varepsilon}^{0} \in\left(\mathbb{R}^{+}\right)^{\mathbb{Z}}, u_{\varepsilon}^{0} \in \mathbb{R}^{\mathbb{Z}}$ periodic. Let $k \in\left\{0, \ldots,\left\lfloor\frac{T}{\Delta T}\right\rfloor\right\}$ and $n_{\varepsilon}^{k} \in \mathbb{R}^{\mathbb{Z}}, p_{\varepsilon}^{k} \in \mathbb{R}^{\mathbb{Z}}, u_{\varepsilon}^{k} \in \mathbb{R}^{\mathbb{Z}}$ a solution to $\left(P_{\varepsilon, \Delta t, \Delta x}^{\prime}\right)$. Then the following relation holds for all $k \in\left\{0, \ldots,\left\lfloor\frac{T}{\Delta t}\right\rfloor\right\}, i \in\{0, \ldots, N\}$ :

$$
\begin{aligned}
& \frac{\Delta x}{2 \Delta t}\left(n_{\varepsilon, i-\frac{1}{2}}^{k+1}\left(u_{\varepsilon, i-\frac{1}{2}}^{k+1}\right)^{2}-n_{\varepsilon, i-\frac{1}{2}}^{k}\left(u_{\varepsilon, i-\frac{1}{2}}^{k}\right)^{2}\right)+\frac{1}{2}\left(F_{\varepsilon, i}^{k+1}\left(u_{\varepsilon, i}^{k+1}\right)^{2}-F_{\varepsilon, i-1}^{k+1}\left(u_{\varepsilon, i-1}^{k+1}\right)^{2}\right) \\
& \quad+\frac{1}{\varepsilon} \delta_{\varepsilon, i-\frac{1}{2}}\left(p^{k+1}\right) u_{\varepsilon, i-\frac{1}{2}}^{k+1}+\frac{T_{e}}{\varepsilon} \delta_{\varepsilon, i-\frac{1}{2}}\left(n^{k+1}\right) u_{\varepsilon, i-\frac{1}{2}}^{k+1}=-R_{\varepsilon, i-\frac{1}{2}}^{k+1},
\end{aligned}
$$

where the residual term is given by

$$
\begin{aligned}
R_{\varepsilon, i-\frac{1}{2}}^{k+1}:= & \frac{\Delta x}{\Delta t} \frac{n_{\varepsilon, i-\frac{1}{2}}^{k}\left(u_{\varepsilon, i-\frac{1}{2}}^{k+1}-u_{\varepsilon, i-\frac{1}{2}}^{k}\right)^{2}}{2}-F_{\varepsilon, i}^{k+1}\left(u_{\varepsilon, i}^{k+1}-u_{\varepsilon, i-\frac{1}{2}}^{k+1}\right)^{2} \\
& +F_{\varepsilon, i-1}^{k+1}\left(u_{\varepsilon, i-1}^{k+1}-u_{\varepsilon, i-\frac{1}{2}}^{k+1}\right)^{2} .
\end{aligned}
$$

Moreover, because of the definition of velocity at the interface, one has $R_{\varepsilon, i-\frac{1}{2}}^{k+1} \geq 0$.

Proof. It is a consequence of Lemma A.1 by taking the convex function $s \in \mathbb{R} \mapsto \psi(s)=\frac{s^{2}}{2}$. The non negativity of the residual term, follows from the definition of the velocity at the interfaces of each cell $C_{i-\frac{1}{2}}$. Indeed one has by definition

We therefore deduce that

$$
u_{\varepsilon, i}^{k+1}:=\left\{\begin{array}{l}
u_{\varepsilon, i-\frac{1}{2}}^{k+1} \text { if } F_{\varepsilon, i}^{k} \geq 0, \\
u_{\varepsilon, i+\frac{1}{2}}^{k+1} \text { else. }
\end{array}\right.
$$

$$
\begin{aligned}
R_{\varepsilon, i-\frac{1}{2}}^{k+1}= & \frac{\Delta x}{\Delta t} \frac{n_{\varepsilon, i-\frac{1}{2}}^{k}\left(u_{\varepsilon, i-\frac{1}{2}}^{k+1}-u_{\varepsilon, i-\frac{1}{2}}^{k}\right)^{2}}{2}+\left(F_{\varepsilon, i}^{k+1}\right)^{-}\left(u_{\varepsilon, i+\frac{1}{2}}^{k+1}-u_{\varepsilon, i-\frac{1}{2}}^{k+1}\right)^{2} \\
& +\left(F_{\varepsilon, i-1}^{k+1}\right)^{+}\left(u_{\varepsilon, i-\frac{3}{2}}^{k+1}-u_{\varepsilon, i-\frac{1}{2}}^{k+1}\right)^{2}
\end{aligned}
$$

We obtained a discrete balance for the kinetic energy, but we need a discrete balance for the total energy. Note that in the kinetic energy balance (4.23), we have two non conservative terms: $\frac{1}{\varepsilon} \delta_{i-\frac{1}{2}}\left(p_{\varepsilon}^{k+1}\right) u_{\varepsilon, i-\frac{1}{2}}^{k+1}$ and $\frac{T_{e}}{\varepsilon} \delta_{i-\frac{1}{2}}\left(n_{\varepsilon}^{k+1}\right) u_{\varepsilon, i-\frac{1}{2}}^{k+1}$. When carrying the discrete summation of the kinetic energy balance, the sum corresponding to this term is compensated by the sum of the non conservative term of the discrete pressure equation (4.7) thanks to the duality formula (4.12). In order to get rid of the second term, we shall mimick at the discrete level the formal computation of Section 2.4. We have the following.

Proposition 4.3 (Discrete potential balance). Let $\varepsilon>0$, let $n_{\varepsilon}^{0} \in\left(\mathbb{R}_{*}^{+}\right)^{\mathbb{Z}}, p_{\varepsilon}^{0} \in\left(\mathbb{R}^{+}\right)^{\mathbb{Z}}, u_{\varepsilon}^{0} \in \mathbb{R}^{\mathbb{Z}}$ periodic. Let $k \in\left\{0, \ldots,\left\lfloor\frac{T}{\Delta T}\right\rfloor\right\}$ and $n_{\varepsilon}^{k} \in \mathbb{R}^{\mathbb{Z}}, p_{\varepsilon}^{k} \in \mathbb{R}^{\mathbb{Z}}, u_{\varepsilon}^{k} \in \mathbb{R}^{\mathbb{Z}}$ a solution to $\left(P_{\varepsilon, \Delta t, \Delta x}^{\prime}\right)$. Then the following relation holds for all $k \in\left\{0, \ldots,\left\lfloor\frac{T}{\Delta t}\right\rfloor\right\}, i \in\{0, \ldots, N\}$ :

$$
\begin{aligned}
& \frac{\Delta x}{\Delta t}\left(n_{\varepsilon, i}^{k+1}\left(\ln \left(n_{\varepsilon, i}^{k+1}\right)-1\right)-n_{\varepsilon, i}^{k}\left(\ln \left(n_{\varepsilon, i}^{k}\right)-1\right)\right)+F_{\varepsilon, i+\frac{1}{2}}^{k+1} \ln \left(n_{\varepsilon, i+1}^{k+1}\right)-F_{\varepsilon, i-\frac{1}{2}}^{k+1} \ln \left(n_{\varepsilon, i}^{k+1}\right) \\
& -u_{\varepsilon, i+\frac{1}{2}}^{k+1} \delta_{\varepsilon, i+\frac{1}{2}}\left(n^{k+1}\right)=-D_{\varepsilon, i}^{k+1}
\end{aligned}
$$


where the dissipative term is non negative and given by:

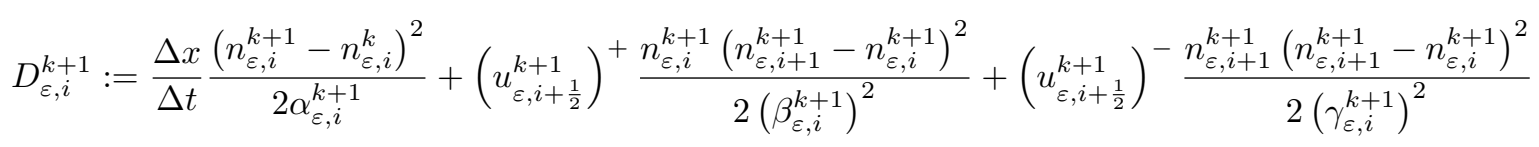

where

$$
\left.\alpha_{\varepsilon, i}^{k+1} \in\right] \min \left(n_{\varepsilon, i}^{k}, n_{\varepsilon, i}^{k+1}\right), \max \left(n_{\varepsilon, i}^{k}, n_{\varepsilon, i}^{k+1}\right)\left[, \beta_{\varepsilon, i}^{k+1}, \gamma_{\varepsilon, i}^{k+1} \in\right] \min \left(n_{\varepsilon, i+1}^{k+1}, n_{\varepsilon, i}^{k+1}\right), \max \left(n_{\varepsilon, i+1}^{k+1}, n_{\varepsilon, i+1}^{k+1}\right)[.
$$

Proof. Let us consider the function $\psi: s \in \mathbb{R}_{+}^{*} \mapsto s(\ln (s)-1)$. Let $k \in\left\{0, \ldots,\left\lfloor\frac{T}{\Delta t}\right\rfloor\right\}$, and $i \in\{0, \ldots, N\}$. Let us multiply the discrete continuity equation $(4.5)$ by $\psi^{\prime}\left(n_{\varepsilon, i}^{k+1}\right)$, therefore we have:

$$
\frac{\Delta x}{\Delta t}\left(n_{\varepsilon, i}^{k+1}-n_{\varepsilon, i}^{k}\right) \psi^{\prime}\left(n_{\varepsilon, i}^{k+1}\right)+\left(F_{\varepsilon, i+\frac{1}{2}}^{k+1}-F_{\varepsilon, i-\frac{1}{2}}^{k+1}\right) \psi^{\prime}\left(n_{\varepsilon, i}^{k+1}\right)=0 .
$$

Since the function $\psi$ is $C^{2}\left(\mathbb{R}_{+}^{*}\right)$ and that the density is everywhere positive, a Taylor-Lagrange expansion around $n_{\varepsilon, i}^{k+1}$ yields the existence of $\left.\alpha_{i}^{k+1} \in\right] \min \left(n_{\varepsilon, i}^{k}, n_{\varepsilon, i}^{k+1}\right), \max \left(n_{\varepsilon, i}^{k}, n_{\varepsilon, i}^{k+1}\right)$ [ such that

$$
\psi\left(n_{\varepsilon, i}^{k}\right)-\psi\left(n_{\varepsilon, i}^{k+1}\right)=\left(n_{\varepsilon, i}^{k}-n_{\varepsilon, i}^{k+1}\right) \psi^{\prime}\left(n_{\varepsilon, i}^{k+1}\right)+\frac{\left(n_{\varepsilon, i}^{k}-n_{\varepsilon, i}^{k+1}\right)^{2} \psi^{\prime \prime}\left(\alpha_{i}^{k}\right)}{2} .
$$

Therefore we can re-write the first term:

$$
\frac{\Delta x}{\Delta t}\left(n_{\varepsilon, i}^{k+1}-n_{\varepsilon, i}^{k}\right) \psi^{\prime}\left(n_{\varepsilon, i}^{k+1}\right)=\frac{\Delta x}{\Delta t}\left(\psi\left(n_{\varepsilon, i}^{k+1}\right)-\psi\left(n_{\varepsilon, i}^{k}\right)\right)+\frac{\left(n_{\varepsilon, i}^{k}-n_{\varepsilon, i}^{k+1}\right)^{2} \psi^{\prime \prime}\left(\alpha_{i}^{k}\right)}{2} .
$$

For the second term, we simply rewrite it as:

$$
\left(F_{\varepsilon, i+\frac{1}{2}}^{k+1}-F_{\varepsilon, i-\frac{1}{2}}^{k+1}\right) \psi^{\prime}\left(n_{\varepsilon, i}^{k+1}\right)=F_{\varepsilon, i+\frac{1}{2}}^{k+1} \psi^{\prime}\left(n_{\varepsilon, i+1}^{k+1}\right)-F_{\varepsilon, i-\frac{1}{2}}^{k+1} \psi^{\prime}\left(n_{\varepsilon, i}^{k+1}\right)+F_{\varepsilon, i+\frac{1}{2}}^{k+1}\left(\psi^{\prime}\left(n_{\varepsilon, i}^{k+1}\right)-\psi^{\prime}\left(n_{\varepsilon, i+1}^{k+1}\right)\right) .
$$

Using the definition of the flux, the expansion of the second term of the right hand side yields

$$
F_{\varepsilon, i+\frac{1}{2}}^{k+1}\left(\psi^{\prime}\left(n_{\varepsilon, i}^{k+1}\right)-\psi^{\prime}\left(n_{\varepsilon, i+1}^{k+1}\right)\right)=n_{\varepsilon, i}^{k+1}\left(\ln \left(n_{\varepsilon, i}^{k+1}\right)-\ln \left(n_{\varepsilon, i+1}^{k+1}\right)\right)\left(u_{\varepsilon, i+\frac{1}{2}}^{k+1}\right)^{+}-n_{\varepsilon, i+1}^{k+1}\left(\ln \left(n_{\varepsilon, i}^{k+1}\right)-\ln \left(n_{\varepsilon, i+1}^{k+1}\right)\right)\left(u_{\varepsilon, i+\frac{1}{2}}^{k+1}\right)^{-} .
$$

since the logarithm function is $C^{2}\left(\mathbb{R}_{+}^{*}\right)$, a Taylor-Lagrange expansion around both $n_{\varepsilon, i}^{k+1}$ and $n_{\varepsilon, i+1}^{k+1}$ yields the existence of $\left.\beta_{i}^{k+1}, \gamma_{i}^{k+1} \in\right] \min \left(n_{\varepsilon, i+1}^{k+1}, n_{\varepsilon, i}^{k+1}\right), \max \left(n_{\varepsilon, i+1}^{k+1}, n_{\varepsilon, i+1}^{k+1}\right)[$ such that:

$$
\begin{aligned}
& \ln \left(n_{\varepsilon, i+1}^{k+1}\right)-\ln \left(n_{\varepsilon, i}^{k+1}\right)=\frac{\left(n_{\varepsilon, i+1}^{k+1}-n_{\varepsilon, i}^{k+1}\right)}{n_{\varepsilon, i}^{k+1}}-\frac{\left(n_{\varepsilon, i+1}^{k+1}-n_{\varepsilon, i}^{k+1}\right)^{2}}{2\left(\beta_{i}^{k+1}\right)^{2}} . \\
& \ln \left(n_{\varepsilon, i}^{k+1}\right)-\ln \left(n_{\varepsilon, i+1}^{k+1}\right)=\frac{\left(n_{\varepsilon, i}^{k+1}-n_{\varepsilon, i+1}^{k+1}\right)}{n_{\varepsilon, i+1}^{k+1}}-\frac{\left(n_{\varepsilon, i}^{k+1}-n_{\varepsilon, i+1}^{k+1}\right)^{2}}{2\left(\gamma_{i}^{k+1}\right)^{2}} .
\end{aligned}
$$

We therefore obtain that

$$
\begin{aligned}
& F_{\varepsilon, i+\frac{1}{2}}^{k+1}\left(\psi^{\prime}\left(n_{\varepsilon, i}^{k+1}\right)-\psi^{\prime}\left(n_{\varepsilon, i+1}^{k+1}\right)\right)=-\left(n_{\varepsilon, i+1}^{k+1}-n_{\varepsilon, i}^{k+1}\right)\left(u_{\varepsilon, i+\frac{1}{2}}^{k+1}\right)^{+}+\frac{n_{\varepsilon, i}^{k+1}\left(n_{\varepsilon, i+1}^{k+1}-n_{\varepsilon, i}^{k+1}\right)^{2}}{2\left(\beta_{i}^{k+1}\right)^{2}}\left(u_{\varepsilon, i+\frac{1}{2}}^{k+1}\right)^{+} \\
& +\left(n_{\varepsilon, i+1}^{k+1}-n_{\varepsilon, i}^{k+1}\right)\left(u_{\varepsilon, i+\frac{1}{2}}^{k+1}\right)^{-}+\frac{n_{\varepsilon, i+1}^{k+1}\left(n_{\varepsilon, i+1}^{k+1}-n_{\varepsilon, i}^{k+1}\right)^{2}}{2\left(\gamma_{i}^{k+1}\right)^{2}}\left(u_{\varepsilon, i+\frac{1}{2}}^{k+1}\right)^{-} .
\end{aligned}
$$


Collecting all the terms together yields

$$
\begin{aligned}
F_{\varepsilon, i+\frac{1}{2}}^{k+1}\left(\psi^{\prime}\left(n_{\varepsilon, i}^{k+1}\right)-\psi^{\prime}\left(n_{\varepsilon, i+1}^{k+1}\right)\right)= & -u_{\varepsilon, i+\frac{1}{2}}^{k+1} \delta_{i+\frac{1}{2}}\left(n_{\varepsilon}^{k+1}\right)+\frac{n_{\varepsilon, i}^{k+1}\left(n_{\varepsilon, i+1}^{k+1}-n_{\varepsilon, i}^{k+1}\right)^{2}}{2\left(\beta_{i}^{k+1}\right)^{2}}\left(u_{\varepsilon, i+\frac{1}{2}}^{k+1}\right)^{+} \\
& +\frac{n_{\varepsilon, i+1}^{k+1}\left(n_{\varepsilon, i+1}^{k+1}-n_{\varepsilon, i}^{k+1}\right)^{2}}{2\left(\gamma_{i}^{k+1}\right)^{2}}\left(u_{\varepsilon, i+\frac{1}{2}}^{k+1}\right)^{-}
\end{aligned}
$$

Gathering all the term together yields the result.

Remark 4.4. Note that the dissipation term $D_{\varepsilon, i}^{k+1}$ does not vanish when $\Delta t$ and $\Delta x$ tend to zero if the density has a discontinuity.

Remark 4.5. The source term $S_{\varepsilon, i}^{k+1}$ is by construction so as $\sum_{i=0}^{N} \frac{S_{\varepsilon, i}^{k+1}}{\gamma-1}-\frac{\varepsilon}{2} R_{\varepsilon, i-\frac{1}{2}}^{k+1}=0$.

We can now prove the following Theorem.

Theorem 4.6 (Existence and unconditional stability).

Let $\varepsilon>0$ and let $n_{\varepsilon}^{0} \in\left(\mathbb{R}_{*}^{+}\right)^{\mathbb{Z}}, p_{\varepsilon}^{0} \in\left(\mathbb{R}^{+}\right)^{\mathbb{Z}}, u_{\varepsilon}^{0} \in \mathbb{R}^{\mathbb{Z}}$ periodic. For all $k \in\left\{0, \ldots,\left\lfloor\frac{T}{\Delta t}\right\rfloor\right\}$ there exists at least one $n_{\varepsilon}^{k} \in\left(\mathbb{R}_{*}^{+}\right)^{\mathbb{Z}}, p_{\varepsilon}^{k} \in\left(\mathbb{R}^{+}\right)^{\mathbb{Z}}, u_{\varepsilon}^{k} \in \mathbb{R}^{\mathbb{Z}}$ solution to $\left(P_{\varepsilon, \Delta t, \Delta x}^{\prime}\right)$. Besides, for all $k \in\left\{0, \ldots,\left\lfloor\frac{T}{\Delta t}\right\rfloor\right\}$ the following holds:

(a)

$$
\sum_{i=0}^{N} n_{\varepsilon, i}^{k}=\sum_{i=0}^{N} n_{\varepsilon, i}^{0}, \quad \sum_{i=0}^{N} n_{\varepsilon, i-\frac{1}{2}}^{k} u_{\varepsilon, i-\frac{1}{2}}^{k}=\sum_{i=0}^{N} n_{\varepsilon, i-\frac{1}{2}}^{0} u_{\varepsilon, i-\frac{1}{2}}^{0}
$$

(b)

$$
\mathcal{E}\left(n_{\varepsilon}^{k+1}, u_{\varepsilon}^{k+1}, p_{\varepsilon}^{k+1}\right)-\mathcal{E}\left(n_{\varepsilon}^{k}, u_{\varepsilon}^{k}, p_{\varepsilon}^{k}\right)=-T_{e} \sum_{i=0}^{N} \Delta t D_{\varepsilon, i}^{k+1}
$$

where $D_{\varepsilon, i}^{n+1} \geq 0$ is defined by (4.25).

(c) Consequently,

$$
-T_{e} \leq \mathcal{E}\left(n_{\varepsilon}^{k}, u_{\varepsilon}^{k}, p_{\varepsilon}^{k}\right) \leq \mathcal{E}\left(n_{\varepsilon}^{0}, u_{\varepsilon}^{0}, p_{\varepsilon}^{0}\right)-T_{e} \sum_{n=0}^{k} \sum_{i=0}^{N} \Delta t D_{\varepsilon, i}^{n+1} .
$$

(d) Moreover, one has

$$
0 \leq \sup _{i=0, . ., N} n_{\varepsilon, i}^{k}+\sum_{i=0}^{N}\left(\frac{\varepsilon n_{\varepsilon, i-\frac{1}{2}}^{k}\left(u_{\varepsilon, i-\frac{1}{2}}^{k}\right)^{2}}{2}+\frac{p_{\varepsilon, i}^{k}}{\gamma-1}\right) \Delta x \leq \frac{\left\|n_{\varepsilon, h}^{0}\right\|}{\Delta x}+\mathcal{E}\left(n_{\varepsilon}^{0}, u_{\varepsilon}^{0}, p_{\varepsilon}^{0}\right)+T_{e} .
$$

(e) Provided the initial data $n_{\varepsilon}^{0}, u_{\varepsilon}^{0}, p_{\varepsilon}^{0}$ is $\mathcal{O}(1)$ as $\varepsilon \rightarrow 0$, we have the $L^{\infty}$-boundedness uniformly in $\varepsilon$ of the density and pressure:

$$
\sup _{i=0, \ldots, N} n_{\varepsilon, i}^{k} \underset{\varepsilon \rightarrow 0}{=} \mathcal{O}(1), \sup _{i=0, . ., N} p_{\varepsilon, i}^{k} \underset{\varepsilon \rightarrow 0}{=} \mathcal{O}(1) .
$$

Proof. We begin with proving the estimates: (a) it suffices to sum the discrete continuity and momentum equation. (b) and (c) We begin with summing the kinetic energy balance (A.1) together with the pressure equation (4.7) (divided by $\gamma-1$ ) and the discrete potential balance (4.25). Then thanks to the periodic boundary 
conditions and the discrete duality between the divergence of the velocity and the gradient of pressure (4.12), the non conservative terms cancel. Moreover, since by construction $\sum_{i=0}^{N} \frac{S_{\varepsilon, i}^{k+1}}{\gamma-1}-\frac{\varepsilon}{2} R_{\varepsilon, i-\frac{1}{2}}^{k+1}=0$, we get

$$
\mathcal{E}\left(n_{\varepsilon}^{n+1}, u_{\varepsilon}^{n+1}, p_{\varepsilon}^{n+1}\right)-\mathcal{E}\left(n_{\varepsilon}^{n}, u_{\varepsilon}^{n}, p_{\varepsilon}^{n}\right)=-T_{e} \sum_{i=0}^{N} \Delta t D_{\varepsilon, i}^{n+1}
$$

where the residual $D_{\varepsilon, i}^{n+1}$ is defined in (4.3). Then it suffices to sum the previous equality from $n=0$ to $n=k-1$ to get the desired result. (d) Note that for all $x \in \mathbb{R}_{*}^{+}, T_{e} x(\ln (x)-1) \geq-T_{e}$ therefore we have, $\sum_{i=0}^{N}\left(\frac{\varepsilon n_{\varepsilon, i-\frac{1}{2}}^{k}\left(u_{\varepsilon, i-\frac{1}{2}}^{k}\right)^{2}}{2}+\frac{p_{\varepsilon, i}^{k}}{\gamma-1}-T_{e}\right) \Delta x \leq \mathcal{E}\left(n_{\varepsilon}^{k}, u_{\varepsilon}^{k}, p_{\varepsilon}^{k}\right)$. Moreover, since $\mathcal{E}\left(n_{\varepsilon}^{k}, u_{\varepsilon}^{k}, p_{\varepsilon}^{k}\right) \leq \mathcal{E}\left(n_{\varepsilon}^{0}, u_{\varepsilon}^{0}, p_{\varepsilon}^{0}\right)$ it comes that

$$
\sum_{i=0}^{N+1}\left(\frac{\varepsilon n_{\varepsilon, i-\frac{1}{2}}^{k}\left(u_{\varepsilon, i-\frac{1}{2}}^{k}\right)^{2}}{2}+\frac{p_{\varepsilon, i}^{k}}{\gamma-1}\right) \Delta x \leq \mathcal{E}\left(n_{\varepsilon}^{0}, u_{\varepsilon}^{0}, p_{\varepsilon}^{0}\right)+T_{e} .
$$

The stability estimate (d) is obtained thanks to the discrete continuity equation (4.5) combined with the conservation of the $L^{1}$-norm and the positivity of the density, one has

$$
0<\sup _{i=0, \ldots, N} n_{\varepsilon, i}^{k} \leq \frac{\sum_{i=0}^{N} n_{\varepsilon, i}^{k} \Delta x}{\Delta x}=\frac{\left\|n_{\varepsilon, h}^{0}\right\|_{L^{1}}}{\Delta x} .
$$

The last estimate (e) is a consequence of (d). For the sake of simplicity, we now omit to precise the dependence on $\varepsilon$ of the discrete density, pressure and velocity. Let us now prove that the scheme is well-defined, we shall apply the Brouwer fixed point theorem. By periodicity, it suffices to prove the existence of the scheme over one period. To do so, let us consider $k \in\left\{0, \ldots,\left\lfloor\frac{T}{\Delta t}\right\rfloor\right\}$ and a solution $\left(n^{k}, u^{k}, p^{k}\right)$. We look for $(n, u, p) \in$ $\mathbb{R}^{N+1} \times \mathbb{R}^{N+1} \times \mathbb{R}^{N+1}$ solution to:

$$
\left\{\begin{array}{l}
\forall i \in\{0, \ldots, N\} \\
\frac{\Delta x}{\Delta t}\left(n_{i}-n_{i}^{k}\right)+F_{i+\frac{1}{2}}(n, u)-F_{i-\frac{1}{2}}(n, u)=0 \\
\frac{\Delta x}{\Delta t}\left(n_{i-\frac{1}{2}} u_{i-\frac{1}{2}}-n_{i-\frac{1}{2}}^{k} u_{i-\frac{1}{2}}^{k}\right)+F_{i}(n, u) u_{i}-F_{i-1}(n, u) u_{i-1}+\frac{1}{\varepsilon} \delta_{i-\frac{1}{2}}\left(p+T_{e} n\right)=0 \\
\frac{\Delta x}{\Delta t}\left(p_{i}-p_{i}^{k}\right)+(u p)_{i+\frac{1}{2}}-(u p)_{i-\frac{1}{2}}+(\gamma-1)\left(p_{i}\right)^{+} \delta_{i}(u)=S_{i}(n, u) \\
n_{0}=n_{N+1}, \quad p_{0}=p_{N+1}, \quad u_{-\frac{1}{2}}=u_{N+\frac{1}{2}}
\end{array}\right.
$$

where

$$
\left\{\begin{array}{l}
F_{i+\frac{1}{2}}(n, u)=n_{i} u_{i+\frac{1}{2}}^{+}-n_{i+1} u_{i-\frac{1}{2}}^{-}, \\
F_{i}=\frac{F_{i-\frac{1}{2}}+F_{i+\frac{1}{2}}}{2}, n_{i-\frac{1}{2}}=\frac{n_{i-1}+n_{i}}{2},
\end{array} \quad, \quad u_{i}:=\left\{\begin{array}{l}
u_{i-\frac{1}{2}} \text { if } F_{i} \geq 0, \\
u_{i+\frac{1}{2}} \text { else. }
\end{array}\right.\right.
$$

and

$$
\begin{gathered}
(\text { up })_{i+\frac{1}{2}}:=\left(u_{i+\frac{1}{2}}\right)^{+} p_{i}-\left(u_{i-\frac{1}{2}}\right)^{-} p_{i+1} \\
\frac{2}{\varepsilon(\gamma-1)} S_{i}(n, u):=\frac{\Delta x}{2 \Delta t} n_{i}^{k}\left(u_{i+\frac{1}{2}}-u_{i+\frac{1}{2}}^{k}\right)^{2}+\frac{\Delta x}{2 \Delta t} n_{i}^{k}\left(u_{i-\frac{1}{2}}-u_{i-\frac{1}{2}}^{k}\right)^{2} \\
+\left[\left(F_{i}(n, u)\right)^{+}+\left(F_{i}(n, u)\right)^{-}\right]\left(u_{i+\frac{1}{2}}-u_{i-\frac{1}{2}}\right)^{2}
\end{gathered}
$$


We shall build $(n, u, p)$ as the fixed point of a certain map. Let us introduce for $M>0, B_{M}:=\{u \in$ $\left.\mathbb{R}^{N+1}:\left(\sum_{i=0}^{N} u_{i-\frac{1}{2}}^{2}\right)^{\frac{1}{2}} \leq M\right\}$ which is a convex and compact subset of $\mathbb{R}^{N+1}$. Let us now denote by $S: u \in$ $B_{M} \mapsto(n(u), p(u)) \in\left(\mathbb{R}^{N+1}\right)_{+}^{*} \times\left(\mathbb{R}^{N+1}\right)_{+} \mapsto u^{*}(n(u), p(u))$ the mapping that associates with a given velocity $u$, the density $n(u)>0$ solution to (4.28), the pressure $p(u) \geq 0$ solution to (4.30) and $u^{*}(n(u), p(u)$ ) solution to (4.29). Note that this map is well-defined since for fixed $u \in B_{M}$, the system of equation on $n(u)$ is linear, invertible and yields $n(u) \in\left(\mathbb{R}_{*}^{+}\right)^{N+1}$, the system of equation on $p(u)$ is also linear, invertible and yields $p(u) \in\left(\mathbb{R}^{+}\right)^{N+1}$ and the equation on $u^{*}(n(u), p(u))$ is explicit. Moreover, the map $S$ is continuous on $B_{M}$ for every $M>0$. It remains to prove that there exists $M>0$ (that depends on the mesh size and the previous state at time $t_{k}$ ) such that $S\left(B_{M}\right) \subset B_{M}$. The discrete total energy balance (that is also valid for the system, given the velocity field $u \in \mathbb{R}^{N+1}$ ) yields the following

$$
\sum_{i=0}^{N+1} u_{i-\frac{1}{2}}^{*}(n(u), p(u))^{2}=\frac{2}{\varepsilon} \sum_{i=0}^{N+1} \frac{\varepsilon n_{i-\frac{1}{2}}(u) u_{i-\frac{1}{2}}^{*}(n(u), p(u))^{2}}{2 n_{i-\frac{1}{2}}(u)} \leq \frac{4}{\varepsilon \inf _{i=0, \ldots, N} n_{i}(u)} \frac{\mathcal{E}^{k}+T_{e}}{\Delta x},
$$

where $\mathcal{E}^{k}$ is the discrete total energy associated with the solution $\left(n^{k}, u^{k}, p^{k}\right)$. To conclude, it suffices to prove that we can find $M>0$ such that $\frac{4}{\varepsilon_{i=0, \ldots, N} n_{i}(u)} \frac{\mathcal{E}^{k}+T_{e}}{\Delta x} \leq M^{2}$. To do so, let us estimate the lower bound $\inf _{i=0, \ldots, N} n_{i}(u)$. One has that $n(u)$ satisfies for all $i \in\{0, \ldots, N\}$ :

$$
n_{i}=n_{i}^{k}-\frac{\Delta t}{\Delta x} n_{i}\left(u_{i+\frac{1}{2}}^{+}+u_{i-\frac{1}{2}}^{-}\right)+\frac{\Delta t}{\Delta x} n_{i+1} u_{i+\frac{1}{2}}^{-}+\frac{\Delta t}{\Delta x} n_{i-1} u_{i-\frac{1}{2}}^{+} .
$$

Since $n(u)$ is positive we infer that

$$
n_{i} \geq n_{i}^{k}-\frac{\Delta t}{\Delta x} n_{i}\left(u_{i+\frac{1}{2}}^{+}+u_{i-\frac{1}{2}}^{-}\right) .
$$

Because $u \in B_{M}$, one has $u_{i+\frac{1}{2}}^{+} \leq\left|u_{i+\frac{1}{2}}\right| \leq M$ and $u_{i-\frac{1}{2}}^{-} \leq\left|u_{i-\frac{1}{2}}\right| \leq M$ and therefore $n_{i} \geq n_{i}^{k}-\frac{2 \Delta t}{\Delta x} n_{i} M$, which gives $n_{i} \geq \frac{n_{i}^{k}}{1+\frac{2 \Delta t}{\Delta x} M}$. Therefore we have the lower bound,

$$
\inf _{i=0, \ldots, N} n_{i}(u) \geq \frac{\inf _{i=0, \ldots, N} n_{i}^{k}}{1+\frac{2 \Delta t}{\Delta x} M} .
$$

It thus yields the control of the $L^{2}$-norm of $S(u)$ :

$$
\sum_{i=0}^{N} u_{i-\frac{1}{2}}^{*}(n(u), p(u))^{2} \leq \frac{4\left(1+\frac{2 \Delta t}{\Delta x} M\right)}{\inf _{i=0, \ldots, N} n_{i}^{k}} \frac{\mathcal{E}^{k}+T_{e}}{\Delta x} .
$$

Then we claim that there exists $M>0$ (large enough) such that $\frac{4\left(1+\frac{2 \Delta t}{\Delta x} M\right)}{\sum_{i=0, \ldots, N} n_{i}^{k}} \frac{\mathcal{E}^{k}+T_{e}}{\Delta x} \leq M^{2}$. In virtue of the Brouwer fixed-point theorem there exists at least one $(n, u, p)$ solution to (4.28)-(4.31). By induction we deduce that the scheme is well-defined for all $k \in\left\{0, \ldots,\left\lfloor\frac{T}{\Delta t}\right\rfloor\right\}$.

Remark 4.7. Provided the initial data are $\mathcal{O}(1)$ as $\varepsilon \rightarrow 0^{+}$, the scheme provides bounds that are uniform in $\varepsilon$ for the pressure and the density. Up to subsequences, the sequences $\left(n_{\varepsilon}^{k}\right),\left(p_{\varepsilon}^{k}\right)$ converges to some limit $\left(n_{0}^{k}\right),\left(p_{0}^{k}\right)$. Nevertheless, it is not enough to conclude the asymptotic consistency of the scheme. We need a uniform estimate on the velocity $\left(u_{\varepsilon}^{k}\right)$. Unfortunately, we have not been able to get more informations on the velocity. In the same spirit, it worth noticing that in the case one has a uniform in $\varepsilon$ estimate for the velocity, the kinetic energy must tend to zero as $\varepsilon \rightarrow 0^{+}$and the source term $S_{\varepsilon, i}^{k+1}$ and the residual term $R_{\varepsilon, i}^{k+1}$ as well. 


\section{AN ITERATIVE LINEAR SCHEME TO SOLVE THE NON LINEAR ONE}

\subsection{Definition of the linear scheme}

Here we propose an iterative procedure to solve the non linear scheme $\left(P_{\varepsilon, \Delta t, \Delta x}^{\prime}\right)$ based on a linear implicit scheme. The reason for introducing such an iterative process lies in the fact that, the non linear scheme requires to solve a genuinely non linear system. Standard Newton-like methods may be very cumbersome and delicate to implement. Our motivation here, is therefore to avoid this specific difficulty by offering an iterative scheme where each iteration requires to determine the solution of a linear scheme that is proven to preserve positivity and is linearly $L^{2}$ stable independently of the small parameter $\varepsilon$. Let us now define our iterative scheme: given $\varepsilon>0$ and $k \in\left\{0, \ldots,\left\lfloor\frac{T}{\Delta t}\right\rfloor\right\}$ and $n_{\varepsilon}^{k} \in\left(\mathbb{R}_{*}^{+}\right)^{\mathbb{Z}}, p_{\varepsilon}^{k} \in\left(\mathbb{R}^{+}\right)^{\mathbb{Z}}, u_{\varepsilon}^{k} \in \mathbb{R}^{\mathbb{Z}}$ a solution to $\left(P_{\varepsilon, \Delta t, \Delta x}^{\prime}\right)$, we define at time $t^{k}$ the iterative scheme $I^{k}\left(P_{\varepsilon, \Delta t \Delta x}^{\prime}\right)$ :

$$
\left\{\begin{array}{l}
\left(n^{-1, k}, p^{-1, k}, u^{-1, k}\right)=\left(n_{\varepsilon}^{k}, p_{\varepsilon}^{k}, u_{\varepsilon}^{k}\right),\left(n^{0, k}, p^{0, k}, u^{0, k}\right)=\left(n_{\varepsilon}^{k}, p_{\varepsilon}^{k}, u_{\varepsilon}^{k}\right), \\
\forall r \in \mathbb{N}, \forall i \in\{0, \ldots, N\}, \\
\frac{\Delta x}{\Delta t}\left(n_{i}^{r+1, k}-n_{\varepsilon, i}^{k}\right)+F_{i+\frac{1}{2}}^{r+1, k}-F_{i-\frac{1}{2}}^{r+1, k}=0, \\
\frac{\Delta x}{\Delta t}\left(n_{i-\frac{1}{2}}^{r, k} u_{i-\frac{1}{2}}^{r+1, k}-n_{\varepsilon, i-\frac{1}{2}}^{k} u_{\varepsilon, i-\frac{1}{2}}^{k}\right)+F_{i}^{r, k} u_{i}^{r, k}-F_{i-1}^{r, k} u_{i-1}^{r, k}+\frac{1}{\varepsilon} \delta_{i-\frac{1}{2}}\left(p^{r+1, k}+T_{e} n^{r+1, k}\right)=0, \\
\frac{\Delta x}{\Delta t}\left(p_{i}^{r+1, k}-p_{\varepsilon, i}^{k}\right)+(u p)_{i+\frac{1}{2}}^{r, k}-(u p)_{i-\frac{1}{2}}^{r, k}+(\gamma-1)\left(p_{i}^{r, k}\right)^{+} \delta_{i}\left(u^{r+1, k}\right)=S_{i}^{r, k}, \\
\forall i \in \mathbb{Z}, \\
n_{i}^{r+1, k}=n_{i+N+1}^{r+1, k}, \\
p_{i}^{r+1, k}=p_{i+N+1}^{r+1, k}, \\
u_{i-\frac{1}{2}}^{r+1, k}=u_{i+N+\frac{1}{2}}^{r+1, k},
\end{array}\right.
$$

where the integer $r$ here refers to the internal number of iterations. To lighten the notation we shall omit in this section the dependence on $\varepsilon$ of the internal iteration $\left(n^{r, k}, p^{r, k}, u^{r, k}\right)$. Provided the density remains positive, at each iteration $r \in \mathbb{N}$, the velocity $u^{r+1, k}$ is defined from equation (5.4) depends implicitly on $p^{r+1, k}$ and $n^{r+1, k}$ but explicitly on the flux. Here the mass flux is defined at each iteration $r \in \mathbb{N}$ by:

$$
F_{i+\frac{1}{2}}^{r+1, k}:=n_{i+\frac{1}{2}}^{r, k} u_{i+\frac{1}{2}}^{r+1, k}-\frac{\left(n_{i+1}^{r, k}-n_{i}^{r, k}\right)}{2}\left|u_{i+\frac{1}{2}}^{r, k}\right|,
$$

where $n_{i+\frac{1}{2}}^{r, k}:=\frac{n_{i}^{r, k}+n_{i+1}^{r, k}}{2}$ and the source term is here given by

$$
\begin{aligned}
\frac{2}{\varepsilon(\gamma-1)} S_{i}^{r, k}:= & \frac{\Delta x}{2 \Delta t} n_{\varepsilon, i}^{k}\left(u_{i+\frac{1}{2}}^{r, k}-u_{i+\frac{1}{2}}^{k}\right)^{2}+\frac{\Delta x}{2 \Delta t} n_{\varepsilon, i}^{k}\left(u_{i-\frac{1}{2}}^{r, k}-u_{i-\frac{1}{2}}^{k}\right)^{2} \\
& +\left[\left(F_{i}^{r, k}\right)^{+}+\left(F_{i}^{r, k}\right)^{-}\right]\left(u_{i+\frac{1}{2}}^{r, k}-u_{i-\frac{1}{2}}^{r, k}\right)^{2} .
\end{aligned}
$$

Other quantities are defined similarly as for the non linear scheme (4). Note that if the sequence $\left(n^{r, k}, p^{r, k}, u^{r, k}\right) \in \mathbb{R}^{\mathbb{Z}} \times \mathbb{R}^{\mathbb{Z}} \times \mathbb{R}^{\mathbb{Z}}$ converges to some $(n, u, p) \in \mathbb{R}^{\mathbb{Z}} \times \mathbb{R}^{\mathbb{Z}} \times \mathbb{R}^{\mathbb{Z}}$ then $(n, u, p)$ solves the non linear scheme $\left(P_{\varepsilon, \Delta t, \Delta x}^{\prime}\right)$ at the iteration $k+1$.

\subsection{Reduction to an elliptic system and positivity conservation}

Let $k \in\left\{0, \ldots,\left\lfloor\frac{T}{\Delta t}\right\rfloor\right\}$, provided the density is positive, each internal iteration $r \in \mathbb{N}$ of $I^{k}\left(P_{\varepsilon, \Delta t \Delta x}^{\prime}\right)$ is equivalent to solve a linear elliptic system of unknown $\left(n^{r+1, k}, p^{r+1, k}\right)$. Then the update of the velocity $u^{r+1, k}$ 
becomes an explicit step. For clarity of exposure, we shall defined some discrete finite difference operators. Namely, for a vector $q^{r} \in \mathbb{R}^{\mathbb{Z}}$, and for $i \in \mathbb{Q}$ the discrete centered Laplacian operator centered at $x_{i}$ is defined by:

$$
\Delta_{i}\left(q^{r}\right):=\delta_{i+\frac{1}{2}}\left(q^{r}\right)-\delta_{i-\frac{1}{2}}\left(q^{r}\right) \text { where } \delta_{i+\frac{1}{2}}\left(q^{r}\right):=q_{i+1}^{r}-q_{i}^{r} .
$$

We also define the the diffusion operators:

$$
\begin{aligned}
\Delta_{i}^{n^{-1}}\left(q^{r}\right) & :=\frac{\delta_{i+\frac{1}{2}}\left(q^{r}\right)}{n_{i+\frac{1}{2}}^{r}}-\frac{\delta_{i-\frac{1}{2}}\left(q^{r}\right)}{n_{i-\frac{1}{2}}^{r}} . \\
\Delta_{i}^{|u|}\left(q^{r}\right) & =\delta_{i+\frac{1}{2}}\left(q^{r}\right)\left|u_{i+\frac{1}{2}}^{r}\right|-\delta_{i-\frac{1}{2}}\left(q^{r}\right)\left|u_{i-\frac{1}{2}}^{r}\right| .
\end{aligned}
$$

We can now re-write the iterative scheme. Thanks to the momentum equation (5.4) one has for all $r \in \mathbb{N}$ and for all $i \in\{0, \ldots, N\}$ :

$$
n_{i-\frac{1}{2}}^{r, k} u_{i-\frac{1}{2}}^{r+1, k}=n_{\varepsilon, i-\frac{1}{2}}^{k} u_{\varepsilon, i-\frac{1}{2}}^{k}-\frac{\Delta t}{\Delta x}\left(F_{i}^{r, k} u_{i}^{r, k}-F_{i-1}^{r, k} u_{i-1}^{r, k}\right)-\frac{\Delta t}{\varepsilon \Delta x} \delta_{i-\frac{1}{2}}\left(p^{r+1, k}+T_{e} n^{r+1, k}\right) .
$$

If $n_{i \pm \frac{1}{2}}^{r, k}>0$ one has:

$$
\delta_{i}\left(u^{r+1, k}\right)=\delta_{i}\left(\frac{n_{\varepsilon}^{k} u_{\varepsilon}^{k}}{n^{r, k}}\right)-\frac{\Delta t}{\Delta x} \Delta_{i}^{-n}\left(F^{r, k} u^{r, k}\right)-\frac{\Delta t}{\Delta x \varepsilon} \Delta_{i}^{n^{-1}}\left(p^{r+1, k}+T_{e} n^{r+1, k}\right) .
$$

where $\delta_{i}\left(\frac{n_{\varepsilon}^{k} u_{\varepsilon}^{k}}{n^{r, k}}\right)=\frac{n_{i+\frac{1}{2}}^{k}}{n_{i+\frac{1}{2}}^{r, k}} u_{i+\frac{1}{2}}^{k}-\frac{n_{i-\frac{1}{2}}^{k}}{n_{i-\frac{1}{2}}^{r, k}} u_{i-\frac{1}{2}}^{k}$. Plugging this expression into the pressure equation (5.5) yields

$$
p_{i}^{r+1, k}-(\gamma-1)\left(p_{i}^{r, k}\right)^{+} \frac{(\Delta t)^{2}}{\varepsilon(\Delta x)^{2}} \Delta_{i}^{n^{-1}}\left(p^{r+1, k}+T_{e} n^{r+1, k}\right)=p_{\varepsilon, i}^{k}+\bar{p}_{i}^{r, k}
$$

with

$$
\bar{p}_{i}^{r, k}=(\gamma-1)\left(p_{i}^{r, k}\right)^{+} \frac{(\Delta t)^{2}}{(\Delta x)^{2}} \Delta_{i}^{n^{-1}}\left(F^{r, k} u^{r, k}\right)-(\gamma-1)\left(p_{i}^{r, k}\right)^{+} \frac{\Delta t}{\Delta x} \delta_{i}\left(\frac{n^{k} u^{k}}{n^{r, k}}\right)-\frac{\Delta t}{\Delta x} \delta_{i}\left((u p)^{r, k}\right)+\frac{\Delta t}{\Delta x} S_{i}^{r, k} .
$$

Eventually using the definition of the flux (5.10), the discrete continuity equation (5.3) rewrites

$$
n_{i}^{r+1, k}-\frac{(\Delta t)^{2}}{\varepsilon(\Delta x)^{2}} \Delta_{i}\left(p^{r+1, k}+T_{e} n^{r+1, k}\right)=n_{\varepsilon, i}^{k}+\bar{n}_{i}^{r, k},
$$

with

$$
\bar{n}_{i}^{r, k}=\frac{\Delta t}{2 \Delta x} \Delta_{i}^{|u|}\left(n^{r, k}\right)-\frac{\Delta t}{\Delta x} \delta_{i}\left(n^{k} u^{k}\right)+\frac{(\Delta t)^{2}}{(\Delta x)^{2}} \Delta_{i}\left(F^{r, k} u^{r, k}\right) .
$$

It thus yields a linear elliptic system of unknown $\left(n^{r+1, k}, p^{r+1, k}\right)$ that writes

$$
\left(L_{\varepsilon}^{r+1, k}\right):\left\{\begin{array}{l}
\forall i \in\{0, \ldots, N\} \\
n_{i}^{r+1, k}-\frac{(\Delta t)^{2}}{\varepsilon(\Delta x)^{2}} \Delta_{i}\left(p^{r+1, k}+T_{e} n^{r+1, k}\right)=n_{\varepsilon, i}^{k}+\bar{n}_{i}^{r, k}, \\
p_{i}^{r+1, k}-(\gamma-1)\left(p_{i}^{r, k}\right)^{+} \frac{(\Delta t)^{2}}{\varepsilon(\Delta x)^{2}} \Delta_{i}^{n^{-1}}\left(p^{r+1, k}+T_{e} n^{r+1, k}\right)=p_{\varepsilon, i}^{k}+\bar{p}_{i}^{r, k}, \\
\quad \text { with } \bar{n}_{i}^{r, k} \text { given by }(5.19), \bar{p}_{i}^{r, k} \text { given by }(5.17), \text { and }, \\
\forall i \in \mathbb{Z}, \\
n_{i}^{r+1, k}=n_{i+(N+1)}^{r+1, k}, \quad p_{i}^{r+1, k}=p_{i+(N+1)}^{r+1, k} .
\end{array}\right.
$$

Provided $n^{r}$ is positive, there exists a unique $\left(n^{r+1, k}, p^{r+1, k}\right) \in \mathbb{R}^{\mathbb{Z}} \times \mathbb{R}^{\mathbb{Z}}$ solution to $\left(L_{\varepsilon}^{r+1, k}\right)$. 
Remark 5.1. In the limit $\varepsilon \rightarrow 0^{+}$, the system $\left(L_{\varepsilon}^{r+1, k}\right)$ degenerates into

$$
\left(L_{0}^{r+1, k}\right):\left\{\begin{array}{l}
\forall i \in\{0, \ldots, N\}, \\
\Delta_{i}\left(p^{r+1, k}+T_{e} n^{r+1, k}\right)=0, \Delta_{i}^{n^{-1}}\left(p^{r+1, k}+T_{e} n^{r+1, k}\right)=0, \\
\forall i \in \mathbb{Z}, \\
n_{i}^{r+1, k}=n_{i+(N+1)}^{r+1, k}, \quad p_{i}^{r+1, k}=p_{i+(N+1)}^{r+1, k}
\end{array}\right.
$$

which is an ill-posed problem since any constant vector is solution. This difficulty is overcomed using a DualityBased decomposition [25] which here consists in the decomposition of the solution into $L_{\varepsilon}^{r+1, k}$ into its mean and fluctuation.

We are now going to prove that the solution to $\left(L_{\varepsilon}^{r+1, k}\right)$ is such that $p^{r+1, k}+T_{e} n^{r+1, k} \in\left(\mathbb{R}_{*}^{+}\right)^{\mathbb{Z}}$. To do so, we shall need the following Lemma.

Lemma 5.2. Let $\varepsilon>0$, let $n_{\varepsilon}^{k} \in\left(\mathbb{R}_{*}^{+}\right)^{\mathbb{Z}}, p_{\varepsilon}^{k} \in\left(\mathbb{R}^{+}\right)^{\mathbb{Z}}, u_{\varepsilon}^{k} \in \mathbb{R}^{\mathbb{Z}}$ periodic. Let $r \in \mathbb{N}$ and $n^{r, k} \in\left(\mathbb{R}_{*}^{+}\right)^{\mathbb{Z}}, p^{r, k} \in$ $\left(\mathbb{R}^{+}\right)^{\mathbb{Z}}, u^{r, k} \in(\mathbb{R})^{\mathbb{Z}}$ a solution to $\left(L_{\varepsilon}^{r, k}\right)$. There exits $\delta^{r, k}>0$ that depends only on $\left(n_{\varepsilon}^{k}, p_{\varepsilon}^{k}, u_{\varepsilon}^{k}, n^{r, k}, p^{r, k}, u^{r, k}\right)$ and $T_{e}>0$ such that if $0<\frac{\Delta t}{\Delta x}<\delta^{r, k}$ then $p_{\varepsilon, i}^{k}+T_{e} n_{\varepsilon, i}^{k}+\bar{p}_{i}^{r, k}+T_{e} \bar{n}_{i}^{r, k}>0$ for all $i \in \mathbb{Z}$.

Proof. Let $\lambda:=\frac{\Delta t}{\Delta x}$, then one decomposes for all $i \in\{0, \ldots, N\}$ the rights hand side (5.19) and (5.17) as:

$$
n_{\varepsilon, i}^{k}+\bar{n}_{i}^{r, k}=n_{\varepsilon, i}^{k}-\bar{n}_{-, i}^{r, k}(\lambda)+\bar{n}_{+, i}^{r, k}(\lambda), \quad p_{\varepsilon, i}^{k}+\bar{p}_{i}^{r, k}=p_{\varepsilon, i}^{k}-\bar{p}_{-, i}^{r, k}(\lambda)+\bar{p}_{+, i}^{r, k}(\lambda),
$$

with

$$
\begin{aligned}
& \bar{n}_{-, i}^{r, k}(\lambda)=\lambda^{2} \Delta_{i}\left(F^{r, k} u^{r, k}\right)^{-}+\lambda\left(\frac{\Delta_{i}^{|u|}\left(n^{r, k}\right)^{-}}{2}+\delta_{i}\left(n^{k} u^{k}\right)^{-}\right), \\
& \bar{p}_{-, i}^{r, k}(\lambda)=\lambda^{2}(\gamma-1)\left(p_{i}^{r, k}\right)^{+} \Delta_{i}^{n^{-1}}\left(F^{r, k} u^{r, k}\right)^{-}+\lambda\left((\gamma-1)\left(p_{i}^{r, k}\right)^{+} \delta_{i}\left(\frac{n^{k} u^{k}}{n^{r, k}}\right)^{+}+\delta_{i}\left((u p)^{r, k}\right)^{+}\right) .
\end{aligned}
$$

and where $\bar{n}_{+, i}^{r, k}(\lambda)=\bar{n}_{i}^{r, k}+\bar{n}_{-, i}^{r, k}(\lambda) \geq 0$ and $\bar{p}_{+, i}^{r, k}(\lambda)=\bar{p}_{i}^{r, k}+\bar{p}_{-, i}^{r, k}(\lambda)+\lambda S_{i}^{r, k} \geq 0$ for all $\lambda \geq 0$. Then combining all the terms together, yields that

$$
p_{\varepsilon, i}^{k}+T_{e} n_{\varepsilon, i}^{k}+\bar{p}_{i}^{r, k}+T_{e} \bar{n}_{i}^{r, k}=p_{\varepsilon, i}^{k}+T_{e} n_{\varepsilon, i}^{k}-\bar{p}_{-, i}^{r, k}(\lambda)-T_{e} \bar{n}_{-, i}^{r, k}(\lambda)+\bar{p}_{+, i}^{r, k}(\lambda)+T_{e} \bar{n}_{+, i}^{r, k}(\lambda) .
$$

Since $\bar{p}_{+, i}^{r, k}(\lambda)+T_{e} \bar{n}_{+, i}^{r, k}(\lambda) \geq 0$, it suffices to prove that the second degree polynomial function $f_{i}^{r, k}: \lambda \mapsto$ $p_{\varepsilon, i}^{k}+T_{e} n_{\varepsilon, i}^{k}-\bar{p}_{-, i}^{r, k}(\lambda)-T_{e} \bar{n}_{-, i}^{r, k}(\lambda)$ is positive on some interval containing zero. Now let us remark that $f_{i}^{r, k}(0)=$ $p_{i}^{k}+T_{e} n_{i}^{k}>0$ therefore by continuity there exists $\lambda_{i}^{r, k}>0$ such that if $0<\lambda<\lambda_{i}^{r, k}$ then $f_{i}^{r, k}(\lambda)>0$. Letting $\delta^{r, k}:=\min _{i \in\{0, \ldots, N\}} \lambda_{i}^{r, k}$ we have $0<\lambda<\delta^{r, k} \Rightarrow p_{\varepsilon, i}^{k}+T_{e} n_{\varepsilon, i}^{k}+\bar{p}_{i}^{r, k}+T_{e} \bar{n}_{i}^{r, k}>0$ for all $i \in\{0, \ldots, N\}$ and by periodicity it holds for all $i \in \mathbb{Z}$.

Remark 5.3. Note that for the previous lemma to hold, it is necessary that $p_{\varepsilon, i}^{k}+T_{e} n_{\varepsilon, i}^{k}>0$ for all $i \in\{0, . ., N\}$. This lemma gives a threshold for the CFL number at each iteration. Namely given $k \in\left\{0, \ldots,\left\lfloor\frac{T}{\Delta t}\right\rfloor\right\}$ and $r \in \mathbb{N}$, one can choose the CFL number as $\frac{\Delta t}{\Delta x} \leq \min _{i \in\{0, \ldots, N\}} \sup \left\{\lambda>0 \mid p_{\varepsilon, i}^{k}+T_{e} n_{\varepsilon, i}^{k}-\bar{p}_{-, i}^{r}(\lambda)-T_{e} \bar{n}_{-, i}^{r}(\lambda)>0\right\}$. Note that the supremum is easily computable since it consists in computing the positive roots of a family of second degree polynomials.

Let us now give a result of positivity. 
Theorem 5.4 (Positivity). Let $\varepsilon>0$, let $n_{\varepsilon}^{k} \in\left(\mathbb{R}_{*}^{+}\right)^{\mathbb{Z}}, p_{\varepsilon}^{k} \in\left(\mathbb{R}^{+}\right)^{\mathbb{Z}}, u_{\varepsilon}^{k} \in \mathbb{R}^{\mathbb{Z}}$ periodic. Let $r \in \mathbb{N}$ and $n^{r} \in\left(\mathbb{R}_{*}^{+}\right)^{\mathbb{Z}}, p^{r} \in\left(\mathbb{R}^{+}\right)^{\mathbb{Z}}, u^{r} \in(\mathbb{R})^{\mathbb{Z}}$ a solution to $\left(L_{\varepsilon}^{r, k}\right)$. There exits $\delta^{r, k}>0$ that depends only on $\left(n_{\varepsilon}^{k}, p_{\varepsilon}^{k}, u_{\varepsilon}^{k}, n^{r}, p^{r}, u^{r}\right)$ and $T_{e}>0$ such that if $0<\frac{\Delta t}{\Delta x}<\delta^{r, k}$ then the solution to $\left(L_{\varepsilon}^{r+1, k}\right)$ verifies $p^{r+1, k}+T_{e} n^{r+1, k} \in\left(\mathbb{R}_{*}^{+}\right)^{\mathbb{Z}}$. Moreover if $\Delta_{i^{*}}\left(p^{r+1, k}\right) \geq 0$ and $\Delta_{j^{*}}^{n^{-1}}\left(n^{r+1, k}\right) \geq 0$ where $i^{*}:=\underset{i \in\{0, \ldots, N\}}{\arg \min n_{i}^{r+1, k}}$ and $j^{*}:=\underset{j \in\{0, \ldots, N\}}{\arg } \min p_{j}^{r+1, k}$ then $n^{r+1, k} \in\left(\mathbb{R}_{*}^{+}\right)^{\mathbb{Z}}$ and $p^{r+1, k} \in\left(\mathbb{R}^{+}\right)^{\mathbb{Z}}$.

Proof. Let $\delta^{r, k}>0$ the one of Lemma 5.2. Let us define for all $i \in \mathbb{Z}, w_{i}^{r+1, k}=p_{i}^{r+1, k}+T_{e} n_{i}^{r+1, k}$. Then one has for all $i \in\{0, \ldots, N\}$,

$$
w_{i}^{r+1, k}-\frac{T_{e}(\Delta t)^{2}}{\varepsilon(\Delta x)^{2}} \Delta_{i}\left(w^{r+1, k}\right)-(\gamma-1)\left(p_{i}^{r, k}\right)^{+} \frac{(\Delta t)^{2}}{\varepsilon(\Delta x)^{2}} \Delta_{i}^{n^{-1}}\left(w^{r+1, k}\right)=p_{\varepsilon, i}^{k}+T_{e} n_{\varepsilon, i}^{k}+\bar{p}_{i}^{r, k}+T_{e} \bar{n}_{i}^{r, k}>0 .
$$

Let us show that $w_{i}^{r+1, k}>0$ for all $i \in\{0, \ldots, N\}$. By contradiction, if it is not the case then let $i^{*}=$ $\underset{i \in\{0, \ldots, N\}}{\arg \min } w_{i}^{r+1, k}$. One has therefore $w_{i^{*}}^{r+1} \leq 0, \Delta_{i^{*}}\left(w^{r+1}\right) \geq 0$ and $\Delta_{i *}^{n^{-1}}\left(w^{r+1}\right) \geq 0$, it therefore gives that

$$
w_{i^{*}}^{r+1}-\frac{T_{e}(\Delta t)^{2}}{\varepsilon(\Delta x)^{2}} \Delta_{i^{*}}\left(w^{r+1}\right)-(\gamma-1)\left(p_{i}^{r}\right)^{+} \frac{(\Delta t)^{2}}{\varepsilon(\Delta x)^{2}} \Delta_{i^{*}}^{n^{-1}}\left(w^{r+1}\right) \leq 0,
$$

and leads to a contradiction. Using a similar maximum principle for the elliptic density equation and the pressure equation, one can prove the positivity of the density and the non negativity of the pressure.

\subsection{Linear $L^{2}$-stability analysis for the iterative scheme}

Let us consider a constant state $\left(n_{0}, u_{0}, p_{0}\right) \in \mathbb{R}_{*}^{+} \times \mathbb{R}_{*}^{+} \times \mathbb{R}^{+}$and note that it is an equilibrium to $\left(P_{\varepsilon, \Delta t, \Delta x}^{\prime}\right)$. Let therefore $k \in\left\{0, \ldots,\left\lfloor\frac{T}{\Delta t}\right\rfloor\right\}$ and assume that the initial guess for the iterative scheme $I_{k}\left(P_{\varepsilon, \Delta t, \Delta x}^{\prime}\right)$ writes for all $i \in \mathbb{Z}$ :

$$
n_{i}^{k}=n_{0}+\tilde{n}_{i}^{k}, \quad u_{i-\frac{1}{2}}^{k}=u_{0}+\tilde{u}_{i-\frac{1}{2}}^{k}, \quad p_{i}^{k}=p_{0}+\tilde{p}_{i}^{k},
$$

where $\left(\tilde{n}^{k}, \tilde{u}^{k}, \tilde{p}^{k}\right) \in \mathbb{R}^{\mathbb{Z}} \times \mathbb{R}^{\mathbb{Z}} \times \mathbb{R}^{\mathbb{Z}}$ is a perturbation of the constante state. Now writing the solution of our iterative scheme $I_{k}\left(P_{\varepsilon, \Delta t, \Delta x}^{\prime}\right)$ as

$$
n_{i}^{r, k}=n_{0}+\tilde{n}_{i}^{r, k}, \quad u_{i-\frac{1}{2}}^{r, k}=u_{0}+\tilde{u}_{i-\frac{1}{2}}^{r, k}, \quad p_{i}^{r, k}=p_{0}+\tilde{p}_{i}^{r, k} .
$$

and neglecting the second order terms, we get that the linearized iterative scheme reads $L I^{k}\left(P_{\varepsilon, \Delta t, \Delta x}^{\prime}\right)$ :

$$
\left\{\begin{array}{l}
\left(\tilde{n}^{0, k}, \tilde{u}^{0, k}, \tilde{p}^{0, k}\right)=\left(\tilde{n}^{k}, \tilde{u}^{k}, \tilde{p}^{k}\right) \\
\forall r \in \mathbb{N}, \quad \forall i \in\{0, \ldots, N\} \\
\frac{\Delta x}{\Delta t}\left(\tilde{n}_{i}^{r, k}-\tilde{n}_{i}^{k}\right)+n_{0} \delta_{i}\left(\tilde{u}^{r+1, k}\right)+u_{0} \delta_{i-\frac{1}{2}}\left(\tilde{n}^{r, k}\right)=0 \\
\frac{\Delta x}{\Delta t}\left(\tilde{p}_{i}^{r+1, k}-\tilde{p}_{i}^{k}\right)+p_{0} \delta_{i}\left(\tilde{u}^{r, k}\right)+u_{0} \delta_{i-\frac{1}{2}}\left(\tilde{p}^{r, k}\right)+(\gamma-1) \delta_{i}\left(\tilde{u}^{r+1, k}\right)=0 \\
\frac{\Delta x}{\Delta t} n_{0}\left(\tilde{u}_{i-\frac{1}{2}}^{r+1, k}-\tilde{u}_{i-\frac{1}{2}}^{k}\right)+n_{0} u_{0} \delta_{i-1}\left(\tilde{u}^{r, k}\right)+\frac{1}{\varepsilon} \delta_{i-\frac{1}{2}}\left(\tilde{p}^{r+1, k}+T_{e} \tilde{n}^{r+1, k}\right)=0 \\
\forall i \in \mathbb{Z}, \\
\tilde{n}_{i+(N+1)}^{r+1, k}=\tilde{n}_{i}^{r+1, k}, \tilde{u}_{i+\left(N+\frac{1}{2}\right)}^{r+1, k}=\tilde{u}_{i-\frac{1}{2}}^{r+1, k}, \tilde{p}_{i+(N+1)}^{r+1, k}=\tilde{p}_{i}^{r+1, k}
\end{array}\right.
$$

Assuming for all $r \in \mathbb{N}$ the Fourier decomposition in $L^{2}[0,1]_{\text {per }}$ :

$$
\tilde{n}_{i}^{r, k}=\sum_{l \in \mathbb{Z}} n_{l}^{r, k} \mathrm{e}^{2 \mathbf{i} \pi l x_{i}}, \tilde{u}_{i-\frac{1}{2}}^{r, k}=\sum_{l \in \mathbb{Z}} u_{l}^{r, k} \mathrm{e}^{2 \mathbf{i} \pi l x_{i-\frac{1}{2}}}, \tilde{p}_{i}^{r, k}=\sum_{l \in \mathbb{Z}} p_{l}^{r, k} \mathrm{e}^{2 \mathbf{i} \pi l x_{i}} \text { with } \mathbf{i}^{2}=-1
$$


and injecting these expression in the linearized iterative scheme yields for all modes of index $l \in \mathbb{Z}$ the linear system

$$
A_{l} X_{l}^{r+1, k}=X_{l}^{k}+B_{l} X_{l}^{r, k} \text { with for } l \in \mathbb{N}, X_{l}^{r, k}=\left(\begin{array}{c}
n_{l}^{r, k} \\
u_{l}^{r, k} \\
p_{l}^{r, k}
\end{array}\right), X_{l}^{k}=\left(\begin{array}{c}
n_{l}^{k} \\
u_{l}^{k} \\
p_{l}^{k}
\end{array}\right),
$$

and

$$
\begin{aligned}
A_{l} & =\left(\begin{array}{ccc}
1 & \mathbf{i} s_{l} n_{0} & 0 \\
\frac{T_{e} \mathbf{i} s_{l} \mathrm{e}^{-\mathbf{i} \pi l \Delta x}}{\varepsilon n_{0}} & 1 & \frac{\mathbf{i} s_{l} \mathrm{e}^{-\mathbf{i} \pi l \Delta x}}{\varepsilon n_{0}} \\
0 & (\gamma-1) p_{0} \mathbf{i} s_{l} & 1
\end{array}\right), \\
B_{l} & =\left(\begin{array}{ccc}
-u_{0} \mathbf{i} s_{l} \mathrm{e}^{-\mathbf{i} \pi l \Delta x} & 0 & 0 \\
0 & -u_{0} \mathbf{i} s_{l} \mathrm{e}^{-\mathbf{i} \pi l \Delta x} & 0 \\
0 & -p_{0} \mathbf{i} s_{l} & -u_{0} \mathbf{i} s_{l} \mathrm{e}^{-\mathbf{i} \pi l \Delta x}
\end{array}\right)
\end{aligned}
$$

where $s_{l}:=2 \frac{\Delta t}{\Delta x} \sin (\pi l \Delta x)$. In view of the Parseval identity, the $L^{2}$ norm of the approximation associated with $L I^{k}\left(P_{\varepsilon, \Delta t, \Delta x}^{\prime}\right)$ is given by

$$
\left\|\tilde{n}_{h}^{r}\right\|_{L^{2}}^{2}=\sum_{l \in \mathbb{Z}}\left|n_{l}^{r}\right|^{2},\left\|\tilde{u}_{h}^{r}\right\|_{L^{2}}^{2}=\sum_{l \in \mathbb{Z}}\left|u_{l}^{r}\right|^{2}, \quad\left\|\tilde{p}_{h}^{r}\right\|_{L^{2}}^{2}=\sum_{l \in \mathbb{Z}}\left|p_{r}^{l}\right|^{2}
$$

We shall then say that the linearized iterative scheme is linearly $L^{2}$ stable if for all $l \in \mathbb{Z}$ the sequence $\left(X_{l}^{r, k}\right)_{r \in \mathbb{N}}$ converges and its limit denoted $X_{l}^{k+1, k}$ satisfies $\left\|X_{l}^{k+1, k}\right\|_{2} \leq\left\|X_{l}^{k}\right\|_{2}$ where $\|\cdot\|_{2}$ is the Euclidian norm on $\mathbb{C}^{3}$. Now to proceed further, it is easy to see that the matrices $A_{l}$ and $A_{l}-B_{l}$ are invertible for all $l \in \mathbb{Z}$. One can therefore prove the following.

Lemma 5.5. Let $\|.\|_{2}$ also denote the subordinate Euclidian norm for matrices. If for all $l \in \mathbb{Z},\left\|A_{l}^{-1} B_{l}\right\|_{2}<1$ and $\left\|\left(A_{l}-B_{l}\right)^{-1}\right\|_{2} \leq 1$ then linearized iterative scheme is $L^{2}$-stable.

Proof. We deduce from the recursive formula (5.20) and the assumption that $\left\|A_{l}^{-1} B_{l}\right\|_{2}<1$ for all $l \in \mathbb{Z}$ and $r \in \mathbb{N}$ one has

$$
X_{l}^{r, k}=\left(A_{l}^{-1} B_{l}\right)^{r} X_{l}^{k}+\left(I_{d}-\left(A_{l}^{-1} B_{l}\right)^{r}\right)\left(I_{d}-\left(A_{l}^{-1} B_{l}\right)\right)^{-1} A_{l}^{-1} X_{l}^{k} .
$$

The sequence $\left(X_{l}^{r, k}\right)_{r \in \mathbb{N}}$ converges to

$$
X_{l}^{k+1, k}=\left(I_{d}-\left(A_{l}^{-1} B_{l}\right)\right)^{-1} A_{l}^{-1} X_{l}^{k}=\left(A_{l}\left(I_{d}-A_{l}^{-1} B_{l}\right)\right)^{-1} X_{l}^{k}=\left(A_{l}-B_{l}\right)^{-1} X_{l}^{k}
$$

and it is easy to conclude.

One can show that $\left\|\left(A_{l}-B_{l}\right)^{-1}\right\|_{2} \leq 1$. It therefore suffices to determine under which condition we have $\left\|A_{l}^{-1} B_{l}\right\|_{2}<1$. A necessary condition is that spectral radius of $A_{l}^{-1} B_{l}$ is lower than one. We thus have the following.

Theorem 5.6 (Asymptotic linear $L^{2}$-stability). Let $\left(n_{0}, u_{0}, p_{0}\right) \in \mathbb{R}_{*}^{+} \times \mathbb{R}_{*}^{+} \times \mathbb{R}^{+}$. Assume there is $0<\delta<1$ such $\frac{p_{0}}{T_{e} n_{0}+(\gamma-1) p_{0}}<1-\delta$ and the CFL condition $\frac{\Delta t}{\Delta x}<\frac{1}{2 u_{0}}$. Therefore there is $\eta>0$ such that for any $0<\varepsilon<\eta$ and for all $l \in \mathbb{Z}$ the matrix $A_{l}^{-1} B_{l}$ has three distinct eigen values of modulus strictly lower than 1 . 
Proof. Standard but tedious computations yields that for all $l \in \mathbb{Z}$ the matrix $A_{l}^{-1} B_{l}$ has three eigen values given by:

$$
\begin{gathered}
\lambda_{ \pm}(\varepsilon)=-\frac{\mathrm{e}^{-\mathbf{i} l \pi \Delta x} s_{l}\left(2 \cdot \mathbf{i} n_{0} u_{0} \varepsilon+s_{l} p_{0} \pm 2 \sqrt{s_{l}^{2}\left(\left(\left(T_{e} n_{0}+p_{0}(\gamma-1)\right) n_{0} \mathrm{e}^{-\mathbf{i} l \pi \Delta x} u_{0}^{2} \varepsilon+1 / 4 p_{0}^{2}\right)+\mathbf{i} \varepsilon n_{0} p_{0} u_{0}\right)}\right)}{2 s_{l}^{2}\left(T_{e} n_{0}+p_{0}(\gamma-1)\right) \mathrm{e}^{-\mathbf{i} \pi l \Delta x}+2 \varepsilon n_{0}}, \\
\lambda_{0}=-\mathbf{i} s_{l} u_{0} \mathrm{e}^{-\mathbf{i} \pi l \Delta x} .
\end{gathered}
$$

Remark that $\lambda_{ \pm}(\varepsilon) \underset{\varepsilon \rightarrow 0}{\rightarrow}-\frac{\left(p_{0} \pm p_{0}\right)}{2\left(T_{e} n_{0}+(\gamma-1) p_{0}\right)}$ and $\left|\lambda_{0}\right|=s_{l} u_{0}$. Therefore there is $\eta>0$ such that for all $0<\varepsilon<\eta$, $\left|\lambda_{+}(\varepsilon)\right|<\frac{p_{0}}{T_{e} n_{0}+(\gamma-1) p_{0}}+\delta<1, \quad\left|\lambda_{-}(\varepsilon)\right|<1$. It then suffices that $s_{l} u_{0}<1$, that is the case under the CFL condition $\frac{\Delta t}{\Delta x}<\frac{1}{2 u_{0}}$.

Remark 5.7. Note that the veracity of the condition: there is $0<\delta<1$ such $\frac{p_{0}}{T_{e} n_{0}+(\gamma-1) p_{0}}<1-\delta$ depends on the kind of physics we consider. Typically, if we write the ionic pressure as $p_{0}=T_{i} n_{0}$ where $T_{i}$ is a ionic reference temperature then the inequality writes: $\frac{1}{\frac{T_{e}}{T_{i}}+(\gamma-1)}<1-\delta$. It therefore depends both on the temperature ratio $\frac{T_{e}}{T_{i}}$ and the value of $\gamma$. In the plasma physics context, fluid models are derived from kinetic ones and $\gamma$ takes the form $\gamma=\frac{2}{d_{v}}+1$ where $d_{v}$ is the dimension of the velocity space. When $d_{v} \geq 2$, the stability essentially depends on the temperature ratio.

Remark 5.8. If one replaces in the previous analysis $u_{0}=0$, one can show that the scheme is unconditionally stable.

\section{Numerical Results}

The aim of this section is to illustrate the main features of our non linear scheme $\left(P_{\varepsilon, \Delta t, \Delta x}^{\prime}\right)$ and to test its ability to be stable and consistent in the drift regime $\varepsilon \ll 1$. We shall explain how we proceed numerically: we fix $N \in \mathbb{N}, T>0$ and take $\Delta x=\frac{1}{N+1}$. For $k \in\left\{0, . .,\left\lfloor\frac{T}{\Delta t}\right\rfloor\right\}$ and $r \in \mathbb{N}$ we define the relative $l^{2}$ error at the $r$-th iteration of the solver $I_{k}\left(P_{\varepsilon, \Delta t, \Delta x}^{\prime}\right)$ by $e_{k}^{r+1, k}:=\frac{\left\|W^{r+1, k}-W^{r, k}\right\|_{2}}{\left\|W^{r, k}\right\|_{2}}$ where we denote $W^{r, k}=\left(n^{r, k}, u^{r, k}, p^{r, k}\right)$ the solution of $I^{k}\left(P_{\varepsilon, \Delta t, \Delta x}^{\prime}\right)$ at iteration $r \in \mathbb{N}$ and where $\|\cdot\|_{2}$ denotes the euclidian norm on $\mathbb{R}^{3(N+1)}$. For each iteration $k \in\left\{0, \ldots,\left\lfloor\frac{T}{\Delta t}\right\rfloor\right\}$, the iterative solver $I_{k}\left(P_{\varepsilon, \Delta t, \Delta x}^{\prime}\right)$ computes at each internal iteration $r \in \mathbb{N}$ the time step according to the CFL condition of Lemma 5.2, that is $\Delta t=\Delta x \min _{i \in\{0, \ldots, N\}} \sup \left\{\lambda>0 \mid p_{\varepsilon, i}^{k}+T_{e} n_{\varepsilon, i}^{k}-\right.$ $\left.\bar{p}_{-, i}^{r, k}(\lambda)-T_{e} \bar{n}_{-, i}^{r, k}(\lambda)>0\right\}$. The iterative scheme stops iterating when there is $r \in \mathbb{N}$ such that $e_{k}^{r+1}<10^{-10}$. In all the numerical test cases presented under, the electron temperature is $T_{e}=1$.

\subsection{Shock speed computation in $\varepsilon=\mathcal{O}(1)$ regime}

Here we test the ability of our scheme to capture the correct shock speed. For this test case, homogeneous Neumann boundary conditions are prescribed on each boundary. We consider the Riemann problem with the initial condition defined for all $x \in[0,1]$ by

$$
n_{0}(x)=1.0, \quad p_{0}(x)=\left(10^{3}-1\right) \mathbf{1}_{x \leq 0.5}(x)+\left(10^{-2}-1\right) \mathbf{1}_{x>0.5}(x), \quad u_{0}(x)=0 .
$$

We discretize the problem with $\Delta x=\frac{1}{512}$ and we choose $\varepsilon=1$ and $\gamma=1.4$. We represent in Figure 1 the exact solution and the approximation at time $T=0.012$ and the evolution of the discrete energy (4.22). We see that the scheme computes the correct shock speed and is slightly diffusive. Refining the mesh size diminishes the diffusion. The energy as expected theoretically is non increasing. 


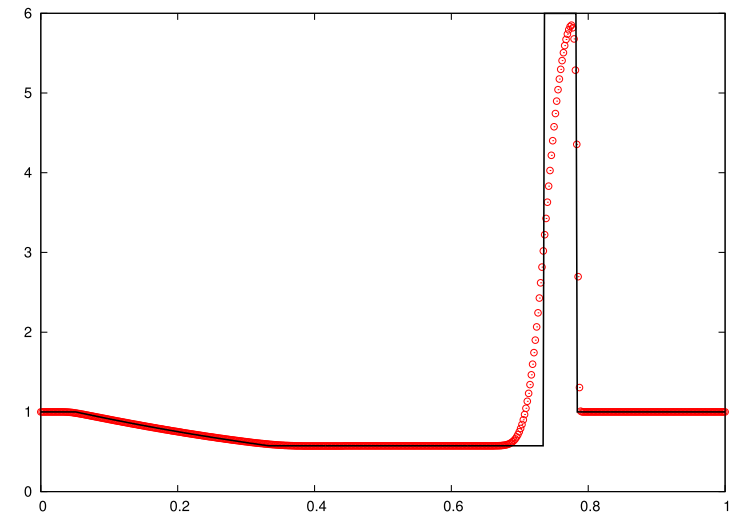

(A) Density

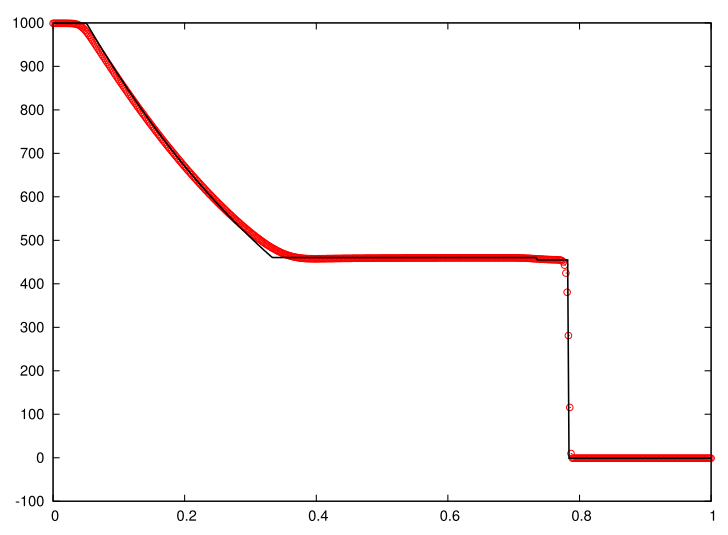

(c) Pressure

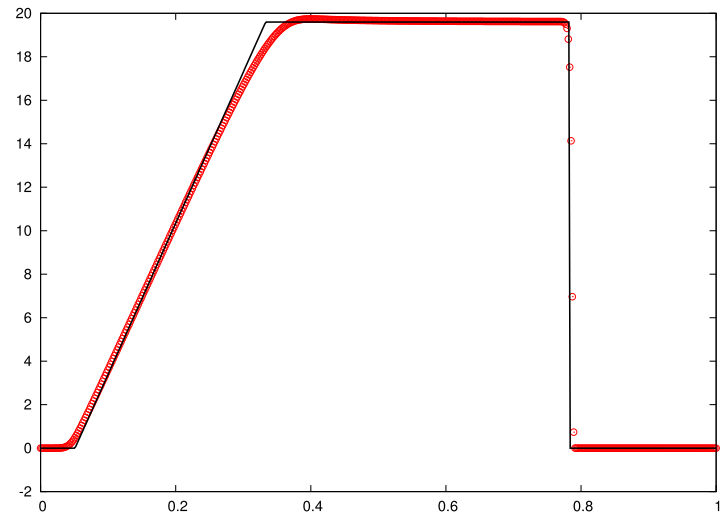

(B) Velocity

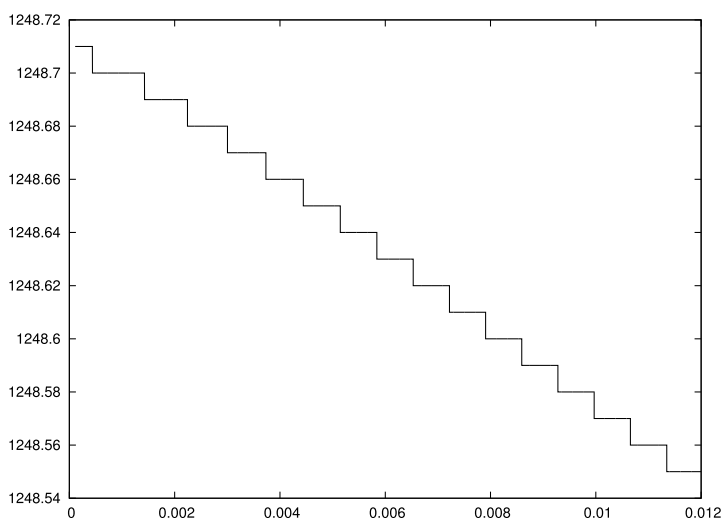

(D) Discrete energy

Figure 1. Riemann problem with initial data (6.1). $\varepsilon=1, T_{e}=1, \gamma=1.4$. The solid line is the exact solution in figure (A),(B) and (C) is the exact solution. Figure (D) is the evolution of the discrete energy.

\subsection{The drift regime $\varepsilon \ll 1$}

For this test case, we consider an initial condition of the form:

$$
\forall x \in[0,1], n_{\varepsilon}^{0}(x)=1+\varepsilon \cos (2 \pi x), u_{\varepsilon}^{0}(x)=1.0, \quad p_{\varepsilon}^{0}(x)=1-\varepsilon \cos (2 \pi x),
$$

with $\gamma=3$. We discretize the problem with a spatial step $\Delta x=1 / 64$ and a temporal step that is fixed $\Delta t=\frac{\Delta x}{100}$, then the CFL is fixed at 0.01 . We look at $\varepsilon$ values of $10^{-4}$ and $10^{-6}$. We compare the numerical solution to $\left(P_{\varepsilon, \Delta t, \Delta x}^{\prime}\right)$ with the analytical solution to $\left(P_{\varepsilon}^{\prime}\right)$ which is given for all $t \geq 0$ and $x \in[0,1]_{\text {per }}$ by $n_{\varepsilon}(t, x)=n_{\varepsilon}^{0}(x-t), u_{\varepsilon}(t, x)=1.0, p_{\varepsilon}(t, x)=p_{\varepsilon}^{0}(x-t)$. In Figure 2, we represent the error between the analytical solution and the numerical approximation given by $\left(P_{\varepsilon, \Delta t, \Delta x}^{\prime}\right)$ at time $T=1.0$. We observe that there is a little error between the limit solution and the numerical approximation. Note that in the case $\varepsilon=10^{-6}$, the CFL $\frac{\Delta t}{\Delta x}$ is ten times greater than $\sqrt{\varepsilon}$ and the scheme is still able to compute a solution which evidences its asymptotic stability. 


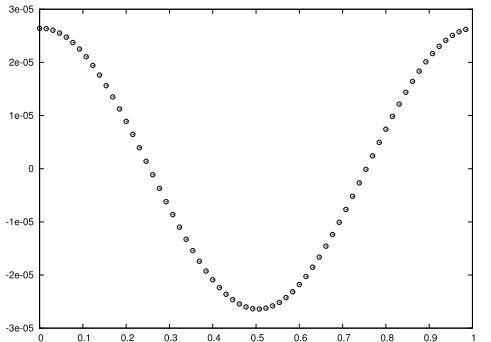

(A) Density error $\varepsilon=10^{-4}$

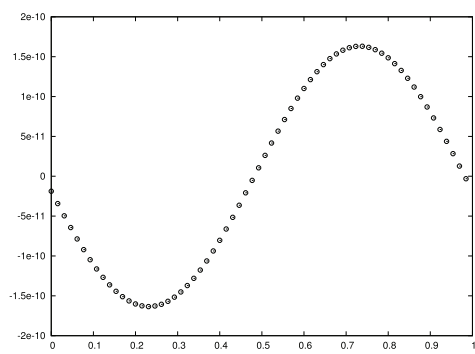

(c) Velocity error $\varepsilon=10^{-4}$

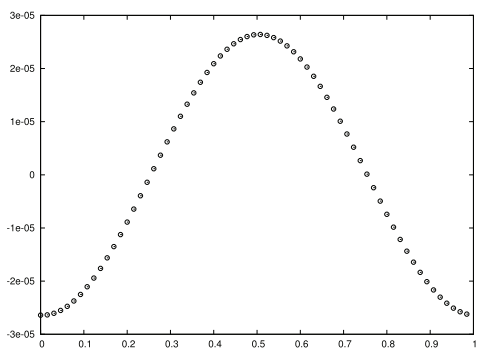

(E) Pressure error $\varepsilon=10^{-4}$

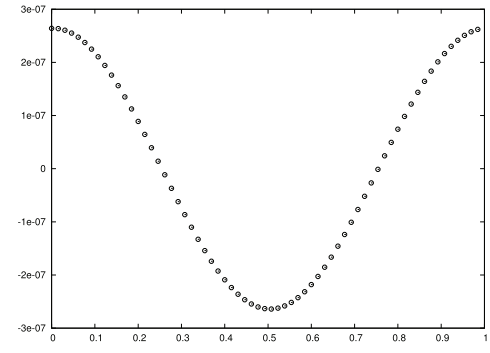

(B) Density error $\varepsilon=10^{-6}$

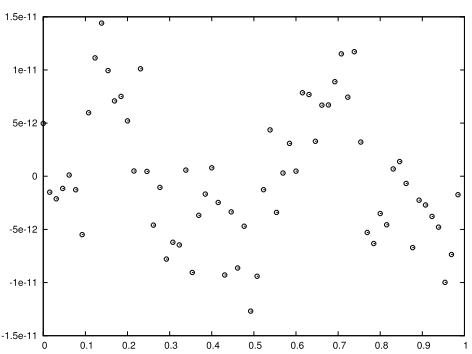

(D) Velocity error $\varepsilon=10^{-6}$

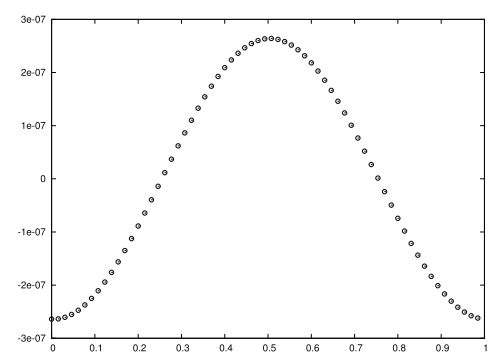

(F) Pressure error $\varepsilon=10^{-6}$

FiguRe 2. Error for the initial (6.2), $T_{e}=1, \gamma=3.0$. Left column: $\varepsilon=10^{-4}$. Right column: $\varepsilon=10^{-6}$. Top row: error on the density. Middle row: error in velocity. Bottom row: error in pressure.

\subsection{Spatial convergence of the scheme}

Here we measure the spatial convergence of $\left(P_{\varepsilon, \Delta t, \Delta x}^{\prime}\right)$ towards an analytical solution. The test case we pay attention to, is a pure transport problem where the velocity remains constant. For $\varepsilon>0$ given, the functions defined for $t \geq 0$ and $x \in[0,1]_{\text {per }}$ by:

$$
n_{\varepsilon}(t, x)=n_{\varepsilon}^{0}(x-t), \quad p_{\varepsilon}(t, x)=p_{\varepsilon}^{0}(x-t), \quad u^{\varepsilon}(t, x)=1.0,
$$

where $p_{\varepsilon}^{0}(x)=1.0+\varepsilon \cos (2 \pi x), n_{\varepsilon}^{0}(x)=\frac{-p_{\varepsilon}^{0}(x)+2.0}{T_{e}}$, and $u_{\varepsilon}^{0}(x)=1$ are solutions to $\left(P_{\varepsilon}^{\prime}\right)$. We perform a spatial convergence study where the CFL is 0.1 . We represent in Figure 3, the total $L^{1}$-error between the analytical solution and its approximation as a function of $\Delta x$ at the final time $T=0.01$ for $\varepsilon \in\left\{1,10^{-2}, 10^{-4}\right\}$. We measure a spatial convergence rate equal to one for all the curves, which is the best we could expect from the scheme since the exact solution is smooth and the flux are first order consistent. 


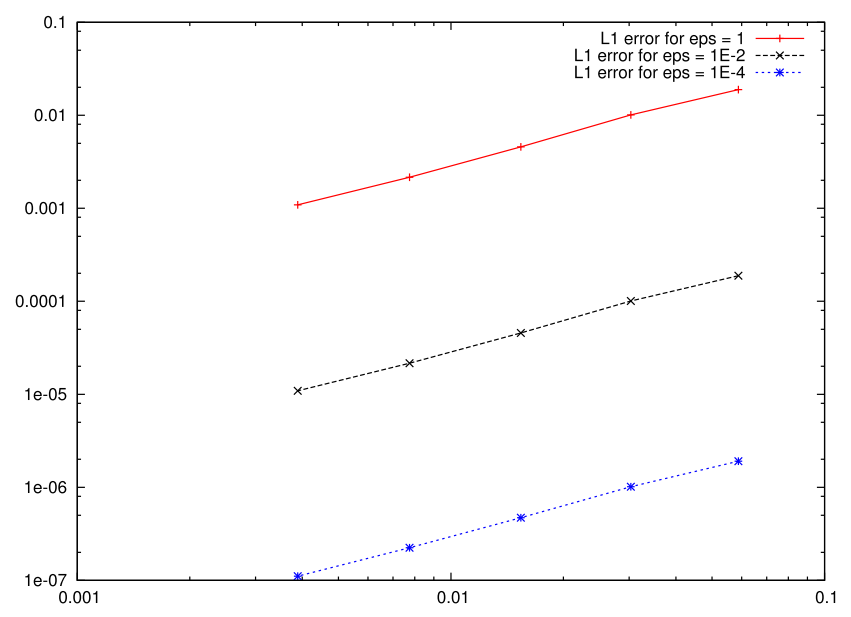

FIGURE 3. $L^{1}$-error (in logarithmic scale) between the exact solution and the numerical solution as a function of $\Delta x$ at the time $T=0.01$ for $\varepsilon \in\left\{1,10^{-2}, 10^{-4}\right\}$. The CFL number is 0.1 .

\section{Conclusion}

We considered the numerical discretization of the quasi-neutral Euler-Boltzmann equations. We proposed a non linear implicit scheme based on staggered grids that was proven to be unconditionally stable and to provide some uniform bounds with respect to $\varepsilon$. Because of its non linearity, we proposed an iterative linear implicit scheme to solve it. The iterative scheme was proven to preserve the positivity and to be $L^{2}$ linearly stable under a CFL condition that does not involve $\varepsilon$. We test the ability of the scheme to compute the correct shock speed through a Riemann problem and we showed that the scheme is stable when the CFL number is larger than $\sqrt{\varepsilon}$. Let us mention some perspectives of amelioration of this work. Due to the lack of uniform estimate for the velocity, the theoretical convergence of the scheme in the drift regime is not proven. This problem is postponed to a future work. Extension of the scheme to higher order in space flux is also a possible perspective. Eventually, the extension of the scheme to a more realistic magnetized plasma model in a three dimensional geometry is an on going work.

\section{Appendix A.}

\section{A.1. Kinetic balance}

We recall a technical lemma needed to derive the kinetic energy balance equation, a proof can be found in [27].

Lemma A.1. Let $\varepsilon>0, T_{e}>0$ and $\psi \in C^{2}(\mathbb{R})$ a convex function. Let $\left(n^{k}, u^{k}, p^{k}\right)_{k \in \mathbb{N}}$ solution to the non linear scheme $\left(P_{\varepsilon, \Delta t, \Delta}^{\prime}\right)$. Then the following relation holds for all $k \in \mathbb{N}$ and $i \in\{0, \ldots, N\}$ :

$$
\begin{aligned}
& \frac{\Delta x}{\Delta t}\left(\psi\left(u_{i-\frac{1}{2}}^{k+1}\right)-\psi\left(u_{i-\frac{1}{2}}^{k}\right)\right)+F_{i}^{k+1} \psi\left(u_{i}^{k+1}\right)-F_{i-1}^{k+1} \psi\left(u_{i-1}^{k+1}\right)+\frac{1}{\varepsilon}\left(p_{i}^{k+1}-p_{i-1}^{k+1}\right) \psi^{\prime}\left(u_{i-\frac{1}{2}}^{k+1}\right) \\
& =-\frac{T_{e}}{\varepsilon}\left(n_{i}^{k+1}-n_{i-1}^{k+1}\right) \psi^{\prime}\left(u_{i-\frac{1}{2}}^{k+1}\right)-R_{i-\frac{1}{2}}^{k+1},
\end{aligned}
$$


where the residual term is given by

$$
\begin{aligned}
R_{i-\frac{1}{2}}^{k+1}:= & \frac{\Delta x}{\Delta t} \frac{n_{i-\frac{1}{2}}^{k}\left(u_{i-\frac{1}{2}}^{k+1}-u_{i-\frac{1}{2}}^{k}\right)^{2}}{2} \psi^{\prime \prime}\left(\xi_{i-\frac{1}{2}}^{k+1}\right) \\
& -F_{i}^{k+1}\left(u_{i}^{k+1}-u_{i-\frac{1}{2}}^{k+1}\right)^{2} \psi^{\prime \prime}\left(\xi_{i}^{k+1}\right) \\
& +F_{i-1}^{k+1}\left(u_{i-1}^{k+1}-u_{i-\frac{1}{2}}^{k+1}\right)^{2} \psi^{\prime \prime}\left(\xi_{i-1}^{k+1}\right)
\end{aligned}
$$

where

$$
\begin{gathered}
\left.\xi_{i-\frac{1}{2}}^{k+1} \in\right] \min \left(u_{i-\frac{1}{2}}^{k+1}, u_{i-\frac{1}{2}}^{k}\right), \max \left(u_{i-\frac{1}{2}}^{k+1}, u_{i-\frac{1}{2}}^{k}\right)\left[, \quad \xi_{i}^{k+1} \in\right] \min \left(u_{i}^{k+1}, u_{i-\frac{1}{2}}^{k+1}\right), \max \left(u_{i}^{k+1}, u_{i-\frac{1}{2}}^{k+1}\right)[, \\
\left.\xi_{i-1}^{k+1} \in\right] \min \left(u_{i-1}^{k+1}, u_{i-\frac{1}{2}}^{k+1}\right), \max \left(u_{i-1}^{k+1}, u_{i-\frac{1}{2}}^{k+1}\right)[.
\end{gathered}
$$

In particular, since $\psi$ is convex and by definition of the flux, the residual term is non negative, $R_{i-\frac{1}{2}}^{k+1} \geq 0$.

Acknowledgements. The author would like to thank Fabrice Deluzet (Institut des Mathématiques de Toulouse) and acknowledges him for many stimulating discussions and useful comments at the early stage of this work. This work has been done in the frame of a Post-Doctoral research contract, it has received the financial support from the ANR MOONRISE (MOdels, Oscillations and NumeRIcal SchEmes, 2015-2019). Support from the Fédération de Fusion pour la Recherche par confinement magntique (FrFCM) in the frame of the project "APREMADAD: Asymptotic-PREserving methods for MAgnetized Plasma simulations: Drift fluid limit and Anisotropic Diffusion problems" (2017-2018) is acknowledged.

\section{REFERENCES}

[1] K. Asano, On the incompressible limit of the compressible euler equation. Jpn. J. Appl. Math 4 (1987) $455-488$.

[2] M. Badsi, M. Campos Pinto and B. Déspres, A minimization formulation of a bi-kinetic sheath. Kinet. Relat. Mod. 9 (2016) 621-656.

[3] G. Bispen, M. Lukacova-Medvidova, and L. Yelsh, Asymptotic preserving IMEX finite volume schemes for low mach number euler equations with gravitation. J. Comput. Phys. 335 (2017) 222-248.

[4] F.F. Chen, Introduction to Plasma Physics and Controlled Fusion. Springer, Berlin (1984).

[5] P. Crispel, P. Degond and M.-H. Vignal, An asymptotic preserving scheme for the two-fluid euler poisson model in the quasineutral limit. J. Comput. Phys. 223 (2007) 208-234.

[6] P. Degond, F. Deluzet, A. Sangam and M.-H. Vignal, An asymptotic-preserving scheme for the euler equations in a strong magnetic field. J. Comput. Phys. 228 (2009) 3540-3558.

[7] F. Deluzet, M. Ottaviani and S. Possaner, A drift-asymptotic scheme for a fluid description of plasmas in strong magnetic fields. Comput. Phys. Commun. 219 (2017) 164-177.

[8] G. Dimarco, R. Loubère and M.-H. Vignal, Study of a new asymptotic preserving scheme for the euler system in the low mach number limit. SIAM J. Sci. Comput. 39 (2017) A2099-A2128.

[9] T. F., Riemann Solvers and Numerical Methods for Fluid Dynamics. Springer-Verlag, Berlin (1997).

[10] F. Filbet and S. Jin, An asymptotic-preserving scheme for the ES-BGK model of the boltzmann equation. J. Sci. Comput. 46 (2011) 204-224.

[11] L. Gastaldo, R. Herbin and J.-C. Latché, A discretization of the phase mass balance in fractional step algorithms for the drift-flux model. IMA J. Numer. Anal. 31 (2011) 116-146.

[12] D. Grapsas, R. Herbin, W. Kheriji and J.-C. Latché, An unconditionnaly stable staggered pressure correction scheme for the compressible navier-stokes equations. J. Comput. Math. 2 (2016) 51-97.

[13] H. Guillard and C. Viozat, On the behavior of upwind schemes in the low Mach number limit. Comput. Fluids 28 (1999) 63-86.

[14] R. Hazeltine and J. Meiss, Plasma Confinement. Inc. Mineola, New York, NY (2003).

[15] J. Jaack, S. Jin and J.-G. Liu, An all-speed asymptotic-preserving method for the isentropic euler and navier-stokes equations. Commun. Comput. Phys. 12 (2012) 955-980.

[16] S. Jin, Runge-Kutta methods for hyperbolic conservation laws with stiff relaxation terms. J. Comput. Phys. 122 (1995) 51-67.

[17] S. Jin, Efficient asymptotic-preserving (AP) schemes for some multiscale kinetic equations. SIAM J. Sci. Comput. 21 (1999) 441-154. 
[18] T. Kato, The cauchy problem for quasi-linear symmetric hyperbolic systems. Ration. Mech. Anal. 58 (1975) $181-205$.

[19] K. Klainerman and A. Majda, Singular limits of quasilinear hyperbolic systems with large parameters and the incompressible limit of compressible fluids. Commun. Pure Appl. Math. 4 (1981) 481-524.

[20] R.J. Leveque, Numerical Methods for Conservation Laws. Birkhauser, Basel (1992).

[21] R.J. Leveque, Finite-Volume Methods for Hyperbolic Problems. Cambridge University Press, Cambridge (2004).

[22] A. Majda, Compressible Fluid Flow and Systems of Conservation Laws in Several Space Variables. Springer, Berlin (1984).

[23] G. Metivier and S. Schochet, The incompressible limit of the non-isentropic euler equations. Arch. Rational Mech. Anal. 158 (2001) 61-90.

[24] C. Negulescu and S. Possaner, Closure of the strongly-magnetized electron fluid equations in the adiabatic regime. Multi. Model. Simul. 14 (2016) 839-873.

[25] P. Degond, F. Deluzet and C. Negulescu, An asymptotic-preserving scheme for strongly anisotropic problems. Multi. Model. Simul. 8 (2010) 645-666.

[26] P. Degond and M. Tang, All speed scheme for the low mach number limit of the itenstropic euler equations. Commun. Comput. Phys. 10 (2011) 1-31.

[27] R. Herbin, J. Latché and T.T. Nguyen, Consistent explicit staggered schemes for the Euler equations. Part II: The Euler equation. Preprint Hal-00821070 (2013).

[28] S. Brull, P. Degond, F. Deluzet and A. Mouton, An asymptotic-preserving scheme for a bi-fluid Euler-Lorentz model. Kinet. Relat. Mod. 4 (2011) 991-1023.

[29] S. Schochet, The compressible euler equations in a bounded domain: existence of solutions and the incompressible limit. Commun. Math. Phys. 104 (1986) 49-75.

[30] P. Stangeby, The Plasma Boundary of Magnetic Fusion Devices. Institute of Physics Publishing, Bristol (2000).

[31] H. Zakerzadeh, On the mach-uniformity of the lagrange-projection scheme. ESAIM: M2AN 51 (2017) 1343-1366. 